\title{
Synthesis, characterization and antimicrobial screening of hybrid molecules containing quinoline, pyrimidine and morpholine analogues
}

\author{
N C DESAI*, K M RAJPARA, V V JOSHI, H V VAGHANI and H M SATODIYA \\ Division of Medicinal Chemistry, Department of Chemistry (DST-FIST Sponsored Department), \\ Mahatma Gandhi Campus, Maharaja Krishnakumarsinhji Bhavnagar University, Bhavnagar 364002 , India \\ e-mail: dnisheeth@rediffmail.com
}

MS received 14 April 2012; revised 1 October 2012; accepted 6 November 2012

\begin{abstract}
In an attempt to find new bio-active molecules, a series of compounds $N$-(4-(2-chloroquinolin3-yl)-6-(aryl)pyrimidin-2-yl)-2-morpholinoacetamides (5a-l) were synthesized by multistep reactions. Compounds were characterized by IR, NMR and mass spectra. Antimicrobial screening of title compounds (5a-l) was carried out against Gram-positive bacteria (Staphylococcus aureus, Streptococcus pyogenes), Gramnegative bacteria (Escherichia coli, Pseudomonas aeruginosa) and fungi (Candida albicans, Aspergillus niger, Aspergillus clavatus) using serial broth dilution method. On the basis of statistical analysis, it is observed that these compounds gave significant co-relation. Newly synthesized compounds $\mathbf{5 e}, \mathbf{5 f}, \mathbf{5 g}, \mathbf{5 i}$ and $\mathbf{5 l}$ showed significant potency against different microbial strains.
\end{abstract}

Keywords. Quinoline; pyrimidine; morpholine; antibacterial activity; antifungal activity; MIC.

\section{Introduction}

A wide array of microorganisms including bacteria, viruses, protozoa and fungi are becoming resistant to drugs that are used to treat infections. This resistance is a major obstacle to the treatment of infectious diseases worldwide. Faced with the extent of antimicrobial drug resistance and the dwindling number of effective antimicrobial drugs, the World Health Organization (WHO) has considered this situation to be one of the greatest threats to human health. Moreover, problems of multi-drug resistant microorganisms have reached an alarming level in many countries around the world. Therefore, it is necessary to have antimicrobial agents with improved potency. In the present research work, we have incorporated design of new compounds and development of hybrid molecules through the combination of different pharmacophores in one heterocyclic congerners, which may lead to compounds with potential antimicrobial activity.

The quinoline skeleton is often used for the design of many synthetic compounds with diverse pharmaceutical properties. The quinoline ring is endowed with various activities, such as antituberculosis, ${ }^{1}$ antimalarial, ${ }^{2}$ anti-inflammatory, ${ }^{3}$ anticancer, ${ }^{4}$ antibiotic, ${ }^{5}$ antihypertensive, ${ }^{6}$ tyrokinase PDGF-RTK inhibiting agents, ${ }^{7}$ and anti-HIV. ${ }^{8,9}$ Keeping this in view, we have designed

*For correspondence two new series of quinoline derivatives with possibly a new mode of action. The structural similarity concepts have been drawn in figure 1, which explains the structural similarity of our new target compounds with renowned drug chloroquine. Substituted aminopyrimidine nuclei are common in marketed drugs such as antiatherosclerotic aronixil, anti-histaminic thonzylamine, antianxielytic buspirone, antihypertensive minoxidil and prazosin, anti-psoriatic enazadrem, and other medicinally relevant compounds. Some notable biological activity of pyrimidine derivatives includes adenosine receptor antagonists, ${ }^{10}$ kinase inhibitors, ${ }^{11}$ analgesic, ${ }^{12}$ anti-inflammatory, ${ }^{12}$ inhibitors of cyclindependent kinases 1 and $2,{ }^{13}$ calcium channel antagonist, ${ }^{14}$ anti-histaminic ${ }^{15}$ and antitubercular ${ }^{16}$ activities. Promising diverse pharmacological activities are shown by various $N$-functionalized morpholines. They are reported to exert a number of important physiological activities such as antibacterial, antifungal, ${ }^{17}$ platelet aggregation inhibitors, ${ }^{18}$ antiemetic ${ }^{19}$ and antidepressants. ${ }^{20}$ Comparative structure of drugs and reported bio-active quinoline derivatives shown in figure 1 .

As a result of remarkable pharmacological efficiency of quinoline, pyrimidine and morpholine derivatives, our studies have been focused towards the synthesis and bio-evaluation of these derivatives by hybrid approach for the search of new antimicrobials. Earlier, our research group has synthesized quinoline derivatives as potential antimicrobial agents. ${ }^{21,22}$ In continuation 


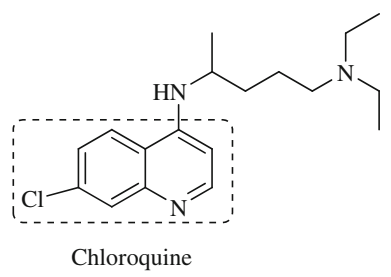

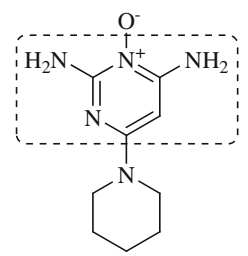

Minoxidil

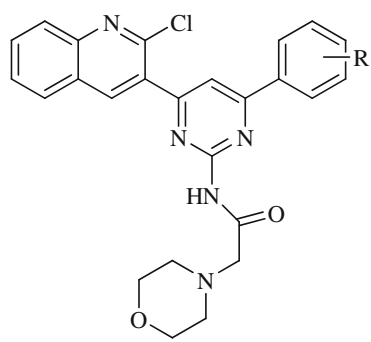

$(5 \mathbf{a}-\mathbf{l})$

Present work

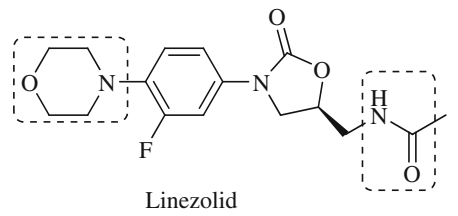

Linezolid

Figure 1. Comparative structure of drugs and reported bio-active quinoline derivatives.

to this, we have synthesized $N$-(4-(2-chloroquinolin3-yl)-6-(aryl)pyrimidin-2-yl)-2-morpholinoacetamides (5a-l) derivatives and screened for their antimicrobial activity. The synthesized compounds (5a-l) were characterized by IR, NMR and mass spectra.

\section{Experimental}

\subsection{Preparation of 2-chloroquinoline-3-carbaldehyde \\ (1)}

To a solution of acetanilide $(5 \mathrm{mmol})$ in dry DMF $(15 \mathrm{mmol})$ at $0-5^{\circ} \mathrm{C}$ with stirring $\mathrm{POCl}_{3}(60 \mathrm{mmol})$ was added drop-wise and the mixture stirred at $100^{\circ} \mathrm{C}$ for time ranging between 4 and $16 \mathrm{~h}$. The mixture was poured into crushed ice, stirred for $5 \mathrm{~min}$ and the resulting solid filtered, washed well with water and dried. The yellow crystals formed were filtered off, washed with water and crystallized from ethyl acetate. ${ }^{23}$

2.2 General procedure for the preparation of 3-(2chloroquinolin-3-yl)-1-(aryl)prop-2-en-1-ones (2a-l)

A mixture of 2-chloroquinoline-3-carbaldehyde (1) $(0.01 \mathrm{~mol})$ and substituted acetophenones $(0.01 \mathrm{~mol})$ was stirred in ethanolic sodium hydroxide for $24 \mathrm{~h}$ at room temperature. The yellow crystals formed were filtered off, washed with water and crystallized from ethanol $(95 \%){ }^{24}$
2.3 General procedure for the preparation of 4-(aryl)6-(2-chloroquinolin-3-yl)pyrimidin-2-amine (3a-l)

A mixture of compounds (2a-l) $(0.01 \mathrm{~mol})$ and guanidine nitrate $(0.01 \mathrm{~mol})$ in ethanol $(99.9 \%)$ was refluxed, while a solution of sodium hydroxide $(0.05 \mathrm{~mol})$ in water was added portion-wise for $2 \mathrm{~h}$. Refluxing was continued for a further $10 \mathrm{~h}$ and the mixture was poured into ice-cold water. The formed solid was separated by filtration. The crude product was dried and crystallized from ethanol (95\%).

2.3a 4-(2-Chloroquinolin-3-yl)-6-phenylpyrimidin-2amine (3a): Light brown crystals, yield: 70\%; m.p.: $177-179^{\circ} \mathrm{C}$; IR (KBr) $v_{\max } / \mathrm{cm}^{-1}: 3450,3344\left(\mathrm{NH}_{2}\right)$, 3070 (C-H, aromatic), $1577(\mathrm{C}=\mathrm{N}), 1515(\mathrm{C}=\mathrm{C}), 725$ $(\mathrm{C}-\mathrm{Cl}) ;{ }^{1} \mathrm{H}$ NMR $\left(300 \mathrm{MHz}, \mathrm{DMSO}-d_{6}, \delta, \mathrm{ppm}\right): 5.65$ (s, $2 \mathrm{H}, \mathrm{NH}_{2}, \mathrm{D}_{2} \mathrm{O}$ exch.), 6.56 (s, $1 \mathrm{H}$, pyrimidine$\mathrm{H}), 7.39-8.54\left(\mathrm{~m}, 10 \mathrm{H}\right.$, Ar-H) ${ }^{13} \mathrm{C}$ NMR $(100 \mathrm{MHz}$, DMSO- $\left.d_{6}, \delta, \mathrm{ppm}\right): 103.5\left(\mathrm{C}_{5}\right), 126.8-143.5(\mathrm{C}-$ arom), 152.3 (C-Cl, quinoline), $163.1\left(\mathrm{C}_{2}\right), 164.8\left(\mathrm{C}_{6}\right)$, $165.2\left(\mathrm{C}_{4}\right)$; $\operatorname{LCMS}(\mathrm{m} / \mathrm{z})$ : $332\left(\mathrm{M}^{+}\right)$; Anal. Calcd. For $\mathrm{C}_{19} \mathrm{H}_{13} \mathrm{ClN}_{4}$ : C-68.57, H-3.94, N-16.84; Found: C-68.64, H-3.88, N-16.76\%.

2.3b 2-(2-Amino-6-(2-chloroquinolin-3-yl)pyrimidin4-yl)phenol (3b): Light yellow crystals, yield: 78\%; m.p.: $193-195^{\circ} \mathrm{C}$; IR (KBr) $v_{\max } / \mathrm{cm}^{-1}: 3417(\mathrm{OH})$, 3452, $3361\left(\mathrm{NH}_{2}\right), 3063(\mathrm{C}-\mathrm{H}$, aromatic), $1570(\mathrm{C}=\mathrm{N})$, $1511(\mathrm{C}=\mathrm{C}), 730(\mathrm{C}-\mathrm{Cl}) ;{ }^{1} \mathrm{H}$ NMR $(300 \mathrm{MHz}, \mathrm{DMSO}-$ $\left.d_{6}, \delta, \mathrm{ppm}\right): 5.60$ (s, $2 \mathrm{H}, \mathrm{NH}_{2}, \mathrm{D}_{2} \mathrm{O}$ exch.), 6.59 (s, $1 \mathrm{H}$, pyrimidine-H), 6.98-8.50 (m, 9H, Ar-H), 9.11 
(s, 1H, OH, $\mathrm{D}_{2} \mathrm{O}$ exch.); ${ }^{13} \mathrm{C}$ NMR (100 MHz, DMSO$\left.d_{6}, \delta, \mathrm{ppm}\right): 103.8$ (C-5), 121.5-149.5 (C-arom), 152.7 (C-Cl, quinoline), 163.4 (C-2), 164.6 (C-6), $165.2(\mathrm{C}-4)$; $\operatorname{LCMS}(\mathrm{m} / \mathrm{z})$ : $348\left(\mathrm{M}^{+}\right)$; Anal. Calcd. For $\mathrm{C}_{19} \mathrm{H}_{13} \mathrm{ClN}_{4} \mathrm{O}$ : C-65.43, H-3.76, N-16.06; Found: C-65.51, H-3.82, N-16.00\%.

2.3c 4-(2-Amino-6-(2-chloroquinolin-3-yl)pyrimidin4-yl)phenol (3c): Light orange crystals, yield: 74\%; m.p.: $167-169^{\circ} \mathrm{C}$; IR (KBr) $v_{\max } / \mathrm{cm}^{-1}: 3411(\mathrm{OH})$, 3440, $3352\left(\mathrm{NH}_{2}\right), 3072(\mathrm{C}-\mathrm{H}$, aromatic), $1575(\mathrm{C}=\mathrm{N})$, $1516(\mathrm{C}=\mathrm{C}), 724(\mathrm{C}-\mathrm{Cl}) ;{ }^{1} \mathrm{H}$ NMR $(300 \mathrm{MHz}, \mathrm{DMSO}-$ $\left.d_{6}, \delta, \mathrm{ppm}\right): 5.62\left(\mathrm{~s}, 2 \mathrm{H}, \mathrm{NH}_{2}, \mathrm{D}_{2} \mathrm{O}\right.$ exch.), 6.62 (s, $1 \mathrm{H}$, pyrimidine- $\mathrm{H}), 6.84-8.48(\mathrm{~m}, 9 \mathrm{H}, \mathrm{Ar}-\mathrm{H}), 9.11$ (s, $1 \mathrm{H}, \mathrm{OH}, \mathrm{D}_{2} \mathrm{O}$ exch.); ${ }^{13} \mathrm{C}$ NMR (100 MHz, DMSO- $d_{6}$, $\delta, \mathrm{ppm}): 104.1$ (C-5), 116.7-156.3 (C-arom), 152.4 (C-Cl, quinoline), 163.1 (C-2), 164.5 (C-6), 165.7 (C-4); LCMS $(\mathrm{m} / z)$ : $348\left(\mathrm{M}^{+}\right)$; Anal. Calcd. For $\mathrm{C}_{19} \mathrm{H}_{13} \mathrm{ClN}_{4} \mathrm{O}$ : C-65.43, H-3.76, N-16.06; Found: C-65.35, H-3.72, N-16.15\%.

2.3d 4-(2-Chloroquinolin-3-yl)-6-(4-methoxyphenyl) pyrimidin-2-amine $(\mathbf{3 d})$ : Light reddish brown crystals, yield: $72 \%$; m.p.: $179-181^{\circ} \mathrm{C}$; IR (KBr) $v_{\max } / \mathrm{cm}^{-1}$ : 3439, $3362\left(\mathrm{NH}_{2}\right), 3067$ (C-H, aromatic), $2925(\mathrm{C}-\mathrm{H}$, $\left.\mathrm{OCH}_{3}\right), 1571(\mathrm{C}=\mathrm{N}), 1520(\mathrm{C}=\mathrm{C}), 740(\mathrm{C}-\mathrm{Cl}) ;{ }^{1} \mathrm{H}$ NMR $\left(300 \mathrm{MHz}, \mathrm{DMSO}-d_{6}, \delta, \mathrm{ppm}\right): 3.68(\mathrm{~s}, 3 \mathrm{H}$, $\left.\mathrm{OCH}_{3}\right), 5.68\left(\mathrm{~s}, 2 \mathrm{H}, \mathrm{NH}_{2}, \mathrm{D}_{2} \mathrm{O}\right.$ exch.), $6.71(\mathrm{~s}, 1 \mathrm{H}$, pyrimidine-H), 7.08-8.45 (m, 9H, Ar-H); ${ }^{13} \mathrm{C}$ NMR $\left(100 \mathrm{MHz}, \mathrm{DMSO}-d_{6}, \delta, \mathrm{ppm}\right): 56.4\left(-\mathrm{OCH}_{3}\right), 104.1$ (C-5), 114.5-160.7 (C-arom), 152.5 (C-Cl, quinoline), 163.7 (C-2), 164.3 (C-6), 165.8 (C-4); LCMS ( $m / z)$ : $362\left(\mathrm{M}^{+}\right)$; Anal. Calcd. For $\mathrm{C}_{20} \mathrm{H}_{15} \mathrm{ClN}_{4} \mathrm{O}$ : C-66.21, H-4.17, N-15.44; Found: C-66.14, H-4.25, N-15.52\%.

2.3e 4-(2-Chlorophenyl)-6-(2-chloroquinolin-3-yl) pyrimidin-2-amine (3e): Light yellow crystals, yield: 70\%; m.p.: $187-189^{\circ} \mathrm{C}$; IR (KBr) $v_{\max } / \mathrm{cm}^{-1}$ : 3443, $3354\left(\mathrm{NH}_{2}\right), 3061(\mathrm{C}-\mathrm{H}$, aromatic $), 1566(\mathrm{C}=\mathrm{N}), 1513$ $(\mathrm{C}=\mathrm{C}), 748(\mathrm{C}-\mathrm{Cl}) ;{ }^{1} \mathrm{H}$ NMR $(300 \mathrm{MHz}$, DMSO$\left.d_{6}, \delta, \mathrm{ppm}\right): 5.72$ (s, 2H, $\mathrm{NH}_{2}, \mathrm{D}_{2} \mathrm{O}$ exch.), 6.69 (s, $1 \mathrm{H}$, pyrimidine- $\mathrm{H}), 7.40-8.48(\mathrm{~m}, 9 \mathrm{H}, \mathrm{Ar}-\mathrm{H}) ;{ }^{13} \mathrm{C}$ NMR $\left(100 \mathrm{MHz}\right.$, DMSO- $\left.d_{6}, \delta, \mathrm{ppm}\right): 103.6$ (C-5), 127.0-144.3 (C-arom), 152.7 (C-Cl, quinoline), 163.3 (C-2), 164.7 (C-6), 165.2 (C-4); LCMS ( $m / z): 366$ $\left(\mathrm{M}^{+}\right)$; Anal. Calcd. For $\mathrm{C}_{19} \mathrm{H}_{12} \mathrm{Cl}_{2} \mathrm{~N}_{4}$ : C-62.14, H-3.29, N-15.26; Found: C-62.22, H-3.37, N-15.34\%.

$2.3 f$ 4-(4-Chlorophenyl)-6-(2-chloroquinolin-3-yl) pyrimidin-2-amine (3f): Yellow crystals, yield: $77 \%$; m.p.: $201-203^{\circ} \mathrm{C}$; IR (KBr) $v_{\max } / \mathrm{cm}^{-1}$ : 3450, 3322
$\left(\mathrm{NH}_{2}\right), 3074$ (C-H, aromatic), $1558(\mathrm{C}=\mathrm{N}), 1514$ $(\mathrm{C}=\mathrm{C}), 751 \quad(\mathrm{C}-\mathrm{Cl}) ;{ }^{1} \mathrm{H}$ NMR $(300 \mathrm{MHz}$, DMSO$\left.d_{6}, \delta, \mathrm{ppm}\right): 5.70$ (s, 2H, $\mathrm{NH}_{2}, \mathrm{D}_{2} \mathrm{O}$ exch.), 6.66 (s, $1 \mathrm{H}$, pyrimidine- $\mathrm{H}), 7.52-8.49(\mathrm{~m}, 9 \mathrm{H}, \mathrm{Ar}-\mathrm{H}) ;{ }^{13} \mathrm{C}$ NMR $\left(100 \mathrm{MHz}, \mathrm{DMSO}-d_{6}, \delta, \mathrm{ppm}\right): 103.6$ (C-5), 127.0-144.3 (C-arom), 152.5 (C-Cl, quinoline), 163.4 (C-2), 164.1 (C-6), 165.7 (C-4); LCMS ( $m / z)$ : 366 $\left(\mathrm{M}^{+}\right)$; Anal. Calcd. For $\mathrm{C}_{19} \mathrm{H}_{12} \mathrm{Cl}_{2} \mathrm{~N}_{4}$ : C-62.14, H-3.29, N-15.26; Found: C-62.06, H-3.22, N-15.32\%.

2.3g 4-(2-Chloroquinolin-3-yl)-6-(2-fluorophenyl) pyrimidin-2-amine $(\mathbf{3 g})$ : Light grey crystals, yield: 80\%; m.p.: $190-191^{\circ} \mathrm{C}$; IR (KBr) $v_{\max } / \mathrm{cm}^{-1}$ : 3433, $3320\left(\mathrm{NH}_{2}\right), 3056(\mathrm{C}-\mathrm{H}$, aromatic $), 1567(\mathrm{C}=\mathrm{N}), 1523$ $(\mathrm{C}=\mathrm{C}), 1145$ (C-F), $743(\mathrm{C}-\mathrm{Cl}) ;{ }^{1} \mathrm{H}$ NMR $(300 \mathrm{MHz}$, DMSO- $d_{6}, \delta, \mathrm{ppm}$ ): 5.77 (s, $2 \mathrm{H}, \mathrm{NH}_{2}, \mathrm{D}_{2} \mathrm{O}$ exch.), $6.75(\mathrm{~s}, 1 \mathrm{H}$, pyrimidine-H), 7.25-8.50 (m, 9H, Ar-H); ${ }^{13} \mathrm{C}$ NMR (100 MHz, DMSO- $\left.d_{6}, \delta, \mathrm{ppm}\right): 104.2$ (C-5), 113.9-157.5 (C-arom), 153.1 (C-Cl, quinoline), 163.8 (C-2), 164.4 (C-6), 165.2 (C-4); LCMS $(m / z): 350$ $\left(\mathrm{M}^{+}\right)$; Anal. Calcd. For $\mathrm{C}_{19} \mathrm{H}_{12} \mathrm{ClFN}_{4}$ : C-65.06, H-3.45, N-15.97; Found: C-65.10, H-3.51, N-15.90\%.

2.3h 4-(2-Chloroquinolin-3-yl)-6-(3-fluorophenyl) pyrimidin-2-amine $(\mathbf{3 h})$ : Light brown crystals, yield: 77\%; m.p.: $175-176^{\circ} \mathrm{C}$; IR (KBr) $v_{\max } / \mathrm{cm}^{-1}$ : 3443, $3361\left(\mathrm{NH}_{2}\right), 3073(\mathrm{C}-\mathrm{H}$, aromatic), $1573(\mathrm{C}=\mathrm{N}), 1528$ $(\mathrm{C}=\mathrm{C}), 1137$ (C-F), 733 (C-Cl); ${ }^{1} \mathrm{H}$ NMR $(300 \mathrm{MHz}$, DMSO- $\left.d_{6}, \delta, \mathrm{ppm}\right): 5.68$ (s, $2 \mathrm{H}, \mathrm{NH}_{2}, \mathrm{D}_{2} \mathrm{O}$ exch.), 6.79 (s, 1H, pyrimidine-H), 7.15-8.53 (m, 9H, Ar-H); ${ }^{13} \mathrm{C}$ NMR (100 MHz, DMSO- $\left.d_{6}, \delta, \mathrm{ppm}\right): 103.9$ (C-5), 116.4-161.7 (C-arom), 152.8 (C-Cl, quinoline), 163.6 (C-2), 164.8 (C-6), 165.2 (C-4); LCMS ( $m / z): 350$ $\left(\mathrm{M}^{+}\right)$; Anal. Calcd. For $\mathrm{C}_{19} \mathrm{H}_{12} \mathrm{ClFN}_{4}$ : C-65.06, H-3.45, N-15.97; Found: C-65.00, H-3.53, N-16.03\%.

$2.3 \mathrm{i}$ 4-(2-Chloroquinolin-3-yl)-6-(4-fluorophenyl) pyrimidin-2-amine (3i): Light reddish crystals, yield: 78\%; m.p.: $178-180^{\circ} \mathrm{C}$; IR (KBr) $v_{\max } / \mathrm{cm}^{-1}$ : 3452, $3345\left(\mathrm{NH}_{2}\right), 3071(\mathrm{C}-\mathrm{H}$, aromatic $), 1555(\mathrm{C}=\mathrm{N}), 1510$ $(\mathrm{C}=\mathrm{C}), 1145$ (C-F), 743 (C-Cl); ${ }^{1} \mathrm{H}$ NMR (300 MHz, DMSO- $\left.d_{6}, \delta, \mathrm{ppm}\right): 5.72$ (s, $2 \mathrm{H}, \mathrm{NH}_{2}, \mathrm{D}_{2} \mathrm{O}$ exch.), 6.69 (s, 1H, pyrimidine-H), 7.40-8.48 (m, 9H, Ar-H); ${ }^{13} \mathrm{C}$ NMR (100 MHz, DMSO- $\left.d_{6}, \delta, \mathrm{ppm}\right): 104.4$ (C-5), 115.6-163.1 (C-arom), 152.8 (C-Cl, quinoline), 163.2 (C-2), 164.8 (C-6), 165.4 (C-4); LCMS ( $m / z): 350$ $\left(\mathrm{M}^{+}\right)$; Anal. Calcd. For $\mathrm{C}_{19} \mathrm{H}_{12} \mathrm{ClFN}_{4}$ : C-65.06, H-3.45, N-15.97; Found: C-65.14, H-3.51, N-15.88\%.

2.3j 4-(2-Chloroquinolin-3-yl)-6-(2-nitrophenyl) pyrimidin-2-amine $(3 j)$ : Dark yellow crystals, yield: 
73\%; m.p.: $186-188^{\circ} \mathrm{C}$; IR (KBr) $v_{\max } / \mathrm{cm}^{-1}: 3461$, $3331\left(\mathrm{NH}_{2}\right), 3056(\mathrm{C}-\mathrm{H}$, aromatic), $1567(\mathrm{C}=\mathrm{N})$, $1521(\mathrm{C}=\mathrm{C}), 1451,1357\left(\mathrm{NO}_{2}\right), 751(\mathrm{C}-\mathrm{Cl}) ;{ }^{1} \mathrm{H}$ NMR (300 MHz, DMSO- $\left.d_{6}, \delta, \mathrm{ppm}\right): 5.72\left(\mathrm{~s}, 2 \mathrm{H}, \mathrm{NH}_{2}, \mathrm{D}_{2} \mathrm{O}\right.$ exch.), 6.69 (s, 1H, pyrimidine-H), 7.58-8.57 (m, 9H, Ar-H); ${ }^{13} \mathrm{C}$ NMR (100 MHz, DMSO- $d_{6}, \delta$, ppm): 104.4 (C-5), 123.6-149.4 (C-arom), 153.1 (C-Cl, quinoline), 163.6 (C-2), 164.4 (C-6), 165.9 (C-4); LCMS $(m / z)$ : $377\left(\mathrm{M}^{+}\right)$; Anal. Calcd. For $\mathrm{C}_{19} \mathrm{H}_{12} \mathrm{ClN}_{5} \mathrm{O}_{2}$ : C-60.41, H-3.20, N-18.54; Found: C-60.33, H-3.26, N-18.62\%.

2.3k 4-(2-Chloroquinolin-3-yl)-6-(3-nitrophenyl) pyrimidin-2-amine $(\mathbf{3 k})$ : Orange yellow crystals, yield: $75 \%$; m.p.: $211-213^{\circ} \mathrm{C}$; IR (KBr) $v_{\max } / \mathrm{cm}^{-1}$ : 3456, $3321\left(\mathrm{NH}_{2}\right), 3067(\mathrm{C}-\mathrm{H}$, aromatic), $1574(\mathrm{C}=\mathrm{N})$, $1518(\mathrm{C}=\mathrm{C}), 1452,1360\left(\mathrm{NO}_{2}\right), 743(\mathrm{C}-\mathrm{Cl}) ;{ }^{1} \mathrm{H}$ NMR (300 MHz, DMSO- $\left.d_{6}, \delta, \mathrm{ppm}\right): 5.77\left(\mathrm{~s}, 2 \mathrm{H}, \mathrm{NH}_{2}, \mathrm{D}_{2} \mathrm{O}\right.$ exch.), 6.63 (s, 1H, pyrimidine-H), 7.61-8.67 (m, 9H, Ar-H); ${ }^{13} \mathrm{C}$ NMR (100 MHz, DMSO- $d_{6}, \delta$, ppm): 103.8 (C-5), 122.7-148.7 (C-arom), 152.6 (C-Cl, quinoline), 163.4 (C-2), 164.7 (C-6), 165.6 (C-4); LCMS $(m / z)$ : $377\left(\mathrm{M}^{+}\right)$; Anal. Calcd. For $\mathrm{C}_{19} \mathrm{H}_{12} \mathrm{ClN}_{5} \mathrm{O}_{2}$ : C-60.41, H-3.20, N-18.54; Found: C-60.46, H-3.16, N-18.48\%.

2.31 4-(2-Chloroquinolin-3-yl)-6-(4-nitrophenyl) pyrimidin-2-amine (3l): Yellow crystals, yield: $82 \%$; m.p.: $192-194^{\circ} \mathrm{C}$; IR (KBr) $v_{\max } / \mathrm{cm}^{-1}: 3441,3334$ $\left(\mathrm{NH}_{2}\right), 3076(\mathrm{C}-\mathrm{H}$, aromatic), $1568(\mathrm{C}=\mathrm{N}), 1518$ $(\mathrm{C}=\mathrm{C}), 1459,1348\left(\mathrm{NO}_{2}\right), 740(\mathrm{C}-\mathrm{Cl}) ;{ }^{1} \mathrm{H}$ NMR (300 MHz, DMSO- $\left.d_{6}, \delta, \mathrm{ppm}\right): 5.74\left(\mathrm{~s}, 2 \mathrm{H}, \mathrm{NH}_{2}, \mathrm{D}_{2} \mathrm{O}\right.$ exch.), 6.67 (s, 1H, pyrimidine-H), 7.60-8.53 (m, 9H, Ar-H); ${ }^{13} \mathrm{C}$ NMR $\left(100 \mathrm{MHz}, \mathrm{DMSO}-d_{6}, \delta, \mathrm{ppm}\right): 104.4$ (C-5), 124.2-147.6 (C-arom), 153.4 (C-Cl, quinoline), 163.6 (C-2), 164.3 (C-6), 165.7 (C-4); LCMS $(m / z)$ : $377\left(\mathrm{M}^{+}\right)$; Anal. Calcd. For $\mathrm{C}_{19} \mathrm{H}_{12} \mathrm{ClN}_{5} \mathrm{O}_{2}$ : C-60.41, H-3.20, N-18.54; Found: C-60.47, H-3.11, N-18.61\%.

2.4 General procedure for the preparation of 2-chloro$\mathrm{N}$-(4-(2-chloroquinolin-3-yl)-6-(aryl)pyrimidin-2-yl) acetamides $(\mathbf{4 a}-\mathbf{l})$

Equimolar amount of compounds (3a-l) (0.01 mol) and chloroacetyl chloride $(0.01 \mathrm{~mol})$ in chloroform was refluxed in the presence of catalytic amount of triethylamine for about $12 \mathrm{~h}$. Excess of solvent was removed under reduced pressure and the residue was stirred with water. The crude product was dried and crystallized from ethanol (95\%).

2.4a 2-Chloro-N-(4-(2-chloroquinolin-3-yl)-6phenylpyrimidin-2-yl)acetamide (4a): Light yellow crystals, yield: $70 \%$; m.p.: $197-199^{\circ} \mathrm{C}$; IR (KBr) $v_{\max } / \mathrm{cm}^{-1}: 3350,3237(\mathrm{NH}), 3059(\mathrm{C}-\mathrm{H}$, aromatic), $2860\left(\mathrm{C}-\mathrm{H}, \mathrm{CH}_{2}\right), 1684(\mathrm{C}=\mathrm{O}), 1612(\mathrm{C}=\mathrm{N}), 1578$ $(\mathrm{C}=\mathrm{C}), 767(\mathrm{C}-\mathrm{Cl}) ;{ }^{1} \mathrm{H}$ NMR $\left(300 \mathrm{MHz}, \mathrm{DMSO}-d_{6}\right.$, $\delta$, ppm): $4.11\left(\mathrm{~s}, 2 \mathrm{H}, \mathrm{CH}_{2}\right), 6.92(\mathrm{~s}, 1 \mathrm{H}$, pyrimidine$\mathrm{H}), 7.43-8.50(\mathrm{~m}, 10 \mathrm{H}, \mathrm{Ar}-\mathrm{H}), 9.76\left(\mathrm{~s}, 1 \mathrm{H}, \mathrm{NH}, \mathrm{D}_{2} \mathrm{O}\right.$ exch.); ${ }^{13} \mathrm{C}$ NMR (100 MHz, DMSO- $d_{6}, \delta$, ppm): 60.4 $\left(\mathrm{CH}_{2}\right), 101.2$ (C-5), 126.2-145.6 (C-arom), 153.6 (C$\mathrm{Cl}$, quinoline), 162.3 (C-2), 164.1 (C-6), 165.4 (C-4), $171.2(\mathrm{C}=\mathrm{O})$; LCMS $(\mathrm{m} / \mathrm{z})$ : $408\left(\mathrm{M}^{+}\right)$; Anal. Calcd. For $\mathrm{C}_{21} \mathrm{H}_{14} \mathrm{Cl}_{2} \mathrm{~N}_{4} \mathrm{O}$ : C-61.63, H-3.45, N-13.69; Found: C-61.69, H-3.52, N-13.62\%.

2.4b 2-Chloro-N-(4-(2-chloroquinolin-3-yl)-6-(2hydroxyphenyl)pyrimidin-2-yl)acetamide (4b): Light brown crystals, yield: 70\%; m.p.: 226-228 ${ }^{\circ} \mathrm{C}$; IR (KBr) $v_{\max } / \mathrm{cm}^{-1}: 3418(\mathrm{OH}), 3340,3230(\mathrm{NH}), 3078(\mathrm{C}-$ $\mathrm{H}$, aromatic), $2869\left(\mathrm{C}-\mathrm{H}, \mathrm{CH}_{2}\right), 1686(\mathrm{C}=\mathrm{O}), 1614$ $(\mathrm{C}=\mathrm{N}), 1567(\mathrm{C}=\mathrm{C}), 773(\mathrm{C}-\mathrm{Cl}) ;{ }^{1} \mathrm{H} \mathrm{NMR}(300 \mathrm{MHz}$, DMSO- $\left.d_{6}, \delta, \mathrm{ppm}\right): 4.16\left(\mathrm{~s}, 2 \mathrm{H}, \mathrm{CH}_{2}\right), 6.89(\mathrm{~s}, 1 \mathrm{H}$, pyrimidine-H), 7.00-8.46 (m, 9H, Ar-H), $9.14(\mathrm{~s}, 1 \mathrm{H}$, $\mathrm{OH}, \mathrm{D}_{2} \mathrm{O}$ exch.), 9.76 (s, $1 \mathrm{H}, \mathrm{NH}, \mathrm{D}_{2} \mathrm{O}$ exch.); ${ }^{13} \mathrm{C}$ NMR $\left(100 \mathrm{MHz}, \mathrm{DMSO}-d_{6}, \delta, \mathrm{ppm}\right): 60.3\left(\mathrm{CH}_{2}\right)$, 101.2 (C-5), 117.5-154.3 (C-arom), 153.4 (C-Cl, quinoline), 162.0 (C-2), 164.4 (C-6), 165.1 (C-4), $171.5(\mathrm{C}=\mathrm{O})$; LCMS $(\mathrm{m} / \mathrm{z}): 424\left(\mathrm{M}^{+}\right)$; Anal. Calcd. For $\mathrm{C}_{21} \mathrm{H}_{14} \mathrm{Cl}_{2} \mathrm{~N}_{4} \mathrm{O}_{2}$ : C-59.31, H-3.32, N-13.17; Found: C-59.37, H-3.41, N-13.12\%.

2.4c 2-Chloro-N-(4-(2-chloroquinolin-3-yl)-6-(4hydroxyphenyl)pyrimidin-2-yl)acetamide (4c): Light brown crystals, yield: $71 \%$; m.p.: $168-170^{\circ} \mathrm{C}$; IR (KBr) $v_{\max } / \mathrm{cm}^{-1}: 3424(\mathrm{OH}), 3347,3219(\mathrm{NH}), 3070(\mathrm{C}-$ $\mathrm{H}$, aromatic), $2874\left(\mathrm{C}-\mathrm{H}, \mathrm{CH}_{2}\right), 1680(\mathrm{C}=\mathrm{O}), 1610$ $(\mathrm{C}=\mathrm{N}), 1514(\mathrm{C}=\mathrm{C}), 768(\mathrm{C}-\mathrm{Cl}) ;{ }^{1} \mathrm{H}$ NMR $(300 \mathrm{MHz}$, DMSO- $\left.d_{6}, \delta, \mathrm{ppm}\right): 4.09\left(\mathrm{~s}, 2 \mathrm{H}, \mathrm{CH}_{2}\right), 6.93(\mathrm{~s}, 1 \mathrm{H}$, pyrimidine-H), 6.78-8.50 (m, 9H, Ar-H), $9.11(\mathrm{~s}, 1 \mathrm{H}$, $\mathrm{OH}, \mathrm{D}_{2} \mathrm{O}$ exch.), 9.73 (s, $1 \mathrm{H}, \mathrm{NH}, \mathrm{D}_{2} \mathrm{O}$ exch.); ${ }^{13} \mathrm{C}$ NMR $\left(100 \mathrm{MHz}, \mathrm{DMSO}-d_{6}, \delta, \mathrm{ppm}\right): 60.6\left(\mathrm{CH}_{2}\right)$, 101.1 (C-5), 115.8-157.4 (C-arom), 153.2 (C-Cl, quinoline), 162.4 (C-2), 164.1 (C-6), 165.6 (C-4), $171.7(\mathrm{C}=\mathrm{O})$; LCMS $(m / z): 424\left(\mathrm{M}^{+}\right)$; Anal. Calcd. For $\mathrm{C}_{21} \mathrm{H}_{14} \mathrm{Cl}_{2} \mathrm{~N}_{4} \mathrm{O}_{2}$ : C-59.31, H-3.32, N-13.17; Found: C-59.39, H-3.28, N-13.25\%.

2.4d 2-Chloro-N-(4-(2-chloroquinolin-3-yl)-6-(4methoxyphenyl)pyrimidin-2-yl)acetamide (4d): Light brown crystals, yield: 68\%; m.p.: $177-179^{\circ} \mathrm{C}$; IR (KBr) $v_{\max } / \mathrm{cm}^{-1}: 3360,3238(\mathrm{NH}), 3062(\mathrm{C}-\mathrm{H}$, aromatic), 2876, 2927 (C-H, $\left.\mathrm{CH}_{2}, \mathrm{CH}_{3}\right), 1685(\mathrm{C}=\mathrm{O}), 1613$ $(\mathrm{C}=\mathrm{N}), 1515(\mathrm{C}=\mathrm{C}), 775(\mathrm{C}-\mathrm{Cl}) ;{ }^{1} \mathrm{H}$ NMR $(300 \mathrm{MHz}$, 
DMSO- $\left.d_{6}, \delta, \mathrm{ppm}\right): 3.56\left(\mathrm{~s}, 3 \mathrm{H}, \mathrm{OCH}_{3}\right), 4.13(\mathrm{~s}$, $\left.2 \mathrm{H}, \mathrm{CH}_{2}\right), 6.84(\mathrm{~s}, 1 \mathrm{H}$, pyrimidine- $\mathrm{H}), 7.09-8.50(\mathrm{~m}$, 9H, Ar-H), 9.69 (s, 1H, NH, $\mathrm{D}_{2} \mathrm{O}$ exch.); ${ }^{13} \mathrm{C}$ NMR $\left(100 \mathrm{MHz}, \mathrm{DMSO}-d_{6}, \delta, \mathrm{ppm}\right): 55.3\left(\mathrm{OCH}_{3}\right), 60.1$ $\left(\mathrm{CH}_{2}\right), 101.3$ (C-5), 113.8-160.4 (C-arom), 154.1 (C$\mathrm{Cl}$, quinoline), 162.5 (C-2), 164.0 (C-6), 165.1 (C-4), $171.4(\mathrm{C}=\mathrm{O})$; LCMS $(\mathrm{m} / \mathrm{z}): 438\left(\mathrm{M}^{+}\right)$; Anal. Calcd. For $\mathrm{C}_{22} \mathrm{H}_{16} \mathrm{Cl}_{2} \mathrm{~N}_{4} \mathrm{O}_{2}$ : C-60.15, H-3.67, N-12.75; Found: C-60.21, H-3.71, N-12.80\%.

2.4e 2-Chloro-N-(4-(2-chlorophenyl)-6-(2-chloroquinolin3-yl)pyrimidin-2-yl)acetamide (4e): Light yellow crystals, yield: $72 \%$; m.p.: $199-201^{\circ} \mathrm{C}$; IR (KBr) $v_{\max } / \mathrm{cm}^{-1}: 3361,3237(\mathrm{NH}), 3074(\mathrm{C}-\mathrm{H}$, aromatic), $2869\left(\mathrm{C}-\mathrm{H}, \mathrm{CH}_{2}\right), 1683(\mathrm{C}=\mathrm{O}), 1610(\mathrm{C}=\mathrm{N}), 1534$ $(\mathrm{C}=\mathrm{C}), 756(\mathrm{C}-\mathrm{Cl}) ;{ }^{1} \mathrm{H}$ NMR $\left(300 \mathrm{MHz}, \mathrm{DMSO}-d_{6}\right.$, $\delta, \mathrm{ppm}): 4.20\left(\mathrm{~s}, 2 \mathrm{H}, \mathrm{CH}_{2}\right), 6.88(\mathrm{~s}, 1 \mathrm{H}$, pyrimidine$\mathrm{H}), 7.30-8.55(\mathrm{~m}, 9 \mathrm{H}, \mathrm{Ar}-\mathrm{H}), 9.74\left(\mathrm{~s}, 1 \mathrm{H}, \mathrm{NH}, \mathrm{D}_{2} \mathrm{O}\right.$ exch. $) ;{ }^{13} \mathrm{C}$ NMR $\left(100 \mathrm{MHz}, \mathrm{DMSO}-d_{6}, \delta, \mathrm{ppm}\right): 60.6$ $\left(\mathrm{CH}_{2}\right), 101.1$ (C-5), 127.3-146.1 (C-arom), $153.6(\mathrm{C}-$ $\mathrm{Cl}$, quinoline), 162.2 (C-2), 164.5 (C-6), 165.7 (C-4), $171.4(\mathrm{C}=\mathrm{O})$; LCMS $(\mathrm{m} / \mathrm{z}): 442\left(\mathrm{M}^{+}\right)$; Anal. Calcd. For $\mathrm{C}_{21} \mathrm{H}_{13} \mathrm{Cl}_{3} \mathrm{~N}_{4} \mathrm{O}$ : C-56.84, H-2.95, N-12.63; Found: C-56.78, H-2.88, N-12.71\%.

2.4f 2-Chloro-N-(4-(4-chlorophenyl)-6-(2-chloroquinolin3-yl)pyrimidin-2-yl)acetamide (4f): Light yellow crystals, yield: 67\%; m.p.: $164-166^{\circ} \mathrm{C}$; IR (KBr) $v_{\max } / \mathrm{cm}^{-1}: 3371,3235(\mathrm{NH}), 3079(\mathrm{C}-\mathrm{H}$, aromatic), $2866\left(\mathrm{C}-\mathrm{H}, \mathrm{CH}_{2}\right), 1684(\mathrm{C}=\mathrm{O}), 1600(\mathrm{C}=\mathrm{N}), 1519$ $(\mathrm{C}=\mathrm{C}), 762(\mathrm{C}-\mathrm{Cl}) ;{ }^{1} \mathrm{H}$ NMR $\left(300 \mathrm{MHz}\right.$, DMSO- $d_{6}$, $\delta$, ppm): $4.29\left(\mathrm{~s}, 2 \mathrm{H}, \mathrm{CH}_{2}\right), 6.92(\mathrm{~s}, 1 \mathrm{H}$, pyrimidine$\mathrm{H}), 7.45-8.53(\mathrm{~m}, 9 \mathrm{H}, \mathrm{Ar}-\mathrm{H}), 9.64\left(\mathrm{~s}, 1 \mathrm{H}, \mathrm{NH}, \mathrm{D}_{2} \mathrm{O}\right.$ exch.); ${ }^{13} \mathrm{C}$ NMR (100 MHz, DMSO- $d_{6}, \delta$, ppm): 60.7 $\left(\mathrm{CH}_{2}\right), 101.5$ (C-5), 127.7-145.7 (C-arom), 153.0 (C$\mathrm{Cl}$, quinoline), 162.7 (C-2), 164.3 (C-6), 165.5 (C-4), $171.9(\mathrm{C}=\mathrm{O})$; LCMS $(\mathrm{m} / z): 442\left(\mathrm{M}^{+}\right)$; Anal. Calcd. For $\mathrm{C}_{21} \mathrm{H}_{13} \mathrm{Cl}_{3} \mathrm{~N}_{4} \mathrm{O}$ : C-56.84, H-2.95, N-12.63; Found: C-56.89, H-2.99, N-12.70\%.

$2.4 \mathrm{~g}$ 2-Chloro-N-(4-(2-chloroquinolin-3-yl)-6-(2fluorophenyl)pyrimidin-2-yl)acetamide $\quad(\mathbf{4 g})$ : Light brown crystals, yield: $71 \%$; m.p.: $182-184^{\circ} \mathrm{C}$; IR (KBr) $v_{\max } / \mathrm{cm}^{-1}: 3351,3242(\mathrm{NH}), 3065(\mathrm{C}-\mathrm{H}$, aromatic), $2865\left(\mathrm{C}-\mathrm{H}, \mathrm{CH}_{2}\right), 1690(\mathrm{C}=\mathrm{O}), 1589(\mathrm{C}=\mathrm{N}), 1523$ $(\mathrm{C}=\mathrm{C}), 1150$ (C-F), $771(\mathrm{C}-\mathrm{Cl}) ;{ }^{1} \mathrm{H}$ NMR $(300 \mathrm{MHz}$, DMSO- $\left.d_{6}, \delta, \mathrm{ppm}\right): 4.28\left(\mathrm{~s}, 2 \mathrm{H}, \mathrm{CH}_{2}\right), 6.94(\mathrm{~s}, 1 \mathrm{H}$, pyrimidine-H), 7.25-8.58 (m, 9H, Ar-H), 9.67 (s, 1H, $\mathrm{NH}, \mathrm{D}_{2} \mathrm{O}$ exch.); ${ }^{13} \mathrm{C}$ NMR $\left(100 \mathrm{MHz}, \mathrm{DMSO}-d_{6}, \delta\right.$, ppm): $60.2\left(\mathrm{CH}_{2}\right), 101.4$ (C-5), 114.7-157.4 (C-arom), 152.6 (C-Cl, quinoline), 162.2 (C-2), 164.5 (C-6),
$165.2(\mathrm{C}-4), 171.4(\mathrm{C}=\mathrm{O})$; LCMS $(m / z): 426\left(\mathrm{M}^{+}\right)$; Anal. Calcd. For $\mathrm{C}_{21} \mathrm{H}_{13} \mathrm{Cl}_{2} \mathrm{FN}_{4} \mathrm{O}$ : C-59.03, H-3.07, N-13.11; Found: C-59.11, H-3.02, N-13.15\%.

2.4h 2-Chloro-N-(4-(2-chloroquinolin-3-yl)-6-(3fluorophenyl)pyrimidin-2-yl)acetamide (4h): Light reddish brown crystals, yield: $68 \%$; m.p.: $156-157^{\circ} \mathrm{C}$; IR $(\mathrm{KBr}) v_{\max } / \mathrm{cm}^{-1}$ : 3355, $3256(\mathrm{NH}), 3070(\mathrm{C}-\mathrm{H}$, aromatic), $2869\left(\mathrm{C}-\mathrm{H}, \mathrm{CH}_{2}\right), 1683(\mathrm{C}=\mathrm{O}), 1581$ $(\mathrm{C}=\mathrm{N}), 1516(\mathrm{C}=\mathrm{C}), 1149(\mathrm{C}-\mathrm{F}), 765(\mathrm{C}-\mathrm{Cl}) ;{ }^{1} \mathrm{H}$ NMR $\left(300 \mathrm{MHz}, \mathrm{DMSO}-d_{6}, \delta, \mathrm{ppm}\right): 4.26\left(\mathrm{~s}, 2 \mathrm{H}, \mathrm{CH}_{2}\right)$, $6.91(\mathrm{~s}, 1 \mathrm{H}$, pyrimidine-H), 7.17-8.52 (m, 9H, Ar-H), 9.73 (s, $1 \mathrm{H}, \mathrm{NH}, \mathrm{D}_{2} \mathrm{O}$ exch.); ${ }^{13} \mathrm{C}$ NMR $(100 \mathrm{MHz}$, DMSO- $\left.d_{6}, \delta, \mathrm{ppm}\right): 60.7\left(\mathrm{CH}_{2}\right), 101.5(\mathrm{C}-5), 114.6-$ 161.7 (C-arom), 153.4 (C-Cl, quinoline), $162.3(\mathrm{C}-2)$, 164.1 (C-6), $165.4(\mathrm{C}-4), 171.5(\mathrm{C}=\mathrm{O})$; LCMS $(\mathrm{m} / \mathrm{z})$ : $426\left(\mathrm{M}^{+}\right)$; Anal. Calcd. For $\mathrm{C}_{21} \mathrm{H}_{13} \mathrm{Cl}_{2} \mathrm{FN}_{4} \mathrm{O}$ : C-59.03, H-3.07, N-13.11; Found: C-59.08, H-3.13, N-13.07\%.

2.4i 2-Chloro-N-(4-(2-chloroquinolin-3-yl)-6-(4fluorophenyl)pyrimidin-2-yl)acetamide (4i): Light brown crystals, yield: 69\%; m.p.: $188-190^{\circ} \mathrm{C}$; IR (KBr) $v_{\max } / \mathrm{cm}^{-1}: 3345,3254(\mathrm{NH}), 3072(\mathrm{C}-\mathrm{H}$, aromatic $)$, $2872\left(\mathrm{C}-\mathrm{H}, \mathrm{CH}_{2}\right), 1690(\mathrm{C}=\mathrm{O}), 1575(\mathrm{C}=\mathrm{N}), 1516$ $(\mathrm{C}=\mathrm{C}), 1154$ (C-F), $776(\mathrm{C}-\mathrm{Cl}) ;{ }^{1} \mathrm{H}$ NMR $(300 \mathrm{MHz}$, DMSO- $\left.d_{6}, \delta, \mathrm{ppm}\right): 4.29\left(\mathrm{~s}, 2 \mathrm{H}, \mathrm{CH}_{2}\right), 6.94(\mathrm{~s}, 1 \mathrm{H}$, pyrimidine-H), 7.34-8.51 (m, 9H, Ar-H), 9.77 (s, 1H, $\mathrm{NH}, \mathrm{D}_{2} \mathrm{O}$ exch.); ${ }^{13} \mathrm{C}$ NMR $\left(100 \mathrm{MHz}, \mathrm{DMSO}-d_{6}, \delta\right.$, ppm): $60.4\left(\mathrm{CH}_{2}\right), 101.5$ (C-5), 115.7-162.6 (C-arom), 152.7 (C-Cl, quinoline), 162.1 (C-2), 164.5 (C-6), 165.7 (C-4), $171.4(\mathrm{C}=\mathrm{O})$; LCMS $(m / z): 426\left(\mathrm{M}^{+}\right)$; Anal. Calcd. For $\mathrm{C}_{21} \mathrm{H}_{13} \mathrm{Cl}_{2} \mathrm{FN}_{4} \mathrm{O}$ : C-59.03, H-3.07, N-13.11; Found: C-58.97, H-3.13, N-13.18\%.

2.4j 2-Chloro-N-(4-(2-chloroquinolin-3-yl)-6-(2nitrophenyl)pyrimidin-2-yl)acetamide (4j): Orange yellow crystals, yield: $66 \%$; m.p.: $167-169^{\circ} \mathrm{C}$; IR (KBr) $v_{\max } / \mathrm{cm}^{-1}: 3365,3283(\mathrm{NH}), 3058(\mathrm{C}-\mathrm{H}$, aromatic), $2853\left(\mathrm{C}-\mathrm{H}, \mathrm{CH}_{2}\right), 1688(\mathrm{C}=\mathrm{O}), 1580(\mathrm{C}=\mathrm{N})$, $1522(\mathrm{C}=\mathrm{C}), 1488,1360\left(\mathrm{NO}_{2}\right), 770(\mathrm{C}-\mathrm{Cl}) ;{ }^{1} \mathrm{H}$ NMR $\left(300 \mathrm{MHz}, \mathrm{DMSO}-d_{6}, \delta, \mathrm{ppm}\right): 4.24\left(\mathrm{~s}, 2 \mathrm{H}, \mathrm{CH}_{2}\right)$, $6.86(\mathrm{~s}, 1 \mathrm{H}$, pyrimidine-H), 7.53-8.57 (m, 9H, Ar-H), 9.73 (s, $1 \mathrm{H}, \mathrm{NH}, \mathrm{D}_{2} \mathrm{O}$ exch.) ${ }^{13} \mathrm{C}$ NMR $(100 \mathrm{MHz}$, DMSO- $\left.d_{6}, \delta, \mathrm{ppm}\right): 60.5\left(\mathrm{CH}_{2}\right), 101.6(\mathrm{C}-5), 124.2-$ 147.4 (C-arom), 152.2 (C-Cl, quinoline), 162.4 (C-2), $164.2(\mathrm{C}-6), 165.8(\mathrm{C}-4), 171.6(\mathrm{C}=\mathrm{O})$; LCMS $(\mathrm{m} / \mathrm{z})$ : $453\left(\mathrm{M}^{+}\right)$; Anal. Calcd. For $\mathrm{C}_{21} \mathrm{H}_{13} \mathrm{Cl}_{2} \mathrm{~N}_{5} \mathrm{O}_{3}$ : C-55.52, H-2.88, N-15.42; Found: C-55.57, H-2.93, N-15.36\%.

$2.4 \mathrm{k} \quad 2$-Chloro-N-(4-(2-chloroquinolin-3-yl)-6-(3nitrophenyl)pyrimidin-2-yl)acetamide $\quad(\mathbf{4 k})$ : Yellow 
crystals, yield: $70 \%$; m.p.: $180-182^{\circ} \mathrm{C}$; IR (KBr) $v_{\max } / \mathrm{cm}^{-1}: 3358,3262(\mathrm{NH}), 3076(\mathrm{C}-\mathrm{H}$, aromatic), $2863\left(\mathrm{C}-\mathrm{H}, \mathrm{CH}_{2}\right), 1692(\mathrm{C}=\mathrm{O}), 1576(\mathrm{C}=\mathrm{N}), 1510$ $(\mathrm{C}=\mathrm{C}), 1485,1358\left(\mathrm{NO}_{2}\right), 785(\mathrm{C}-\mathrm{Cl}) ;{ }^{1} \mathrm{H}$ NMR $\left(300 \mathrm{MHz}, \mathrm{DMSO}-d_{6}, \delta, \mathrm{ppm}\right): 4.25\left(\mathrm{~s}, 2 \mathrm{H}, \mathrm{CH}_{2}\right)$, $6.92(\mathrm{~s}, 1 \mathrm{H}$, pyrimidine-H), 7.52-8.57 (m, 9H, Ar-H), 9.69 (s, 1H, NH, $\mathrm{D}_{2} \mathrm{O}$ exch.); ${ }^{13} \mathrm{C} \mathrm{NMR}(100 \mathrm{MHz}$, DMSO- $\left.d_{6}, \delta, \mathrm{ppm}\right): 60.7\left(\mathrm{CH}_{2}\right), 101.5(\mathrm{C}-5), 103.2-$ 148.3 (C-arom), 152.8 (C-Cl, quinoline), 162.0 (C-2), $164.4(\mathrm{C}-6), 165.9(\mathrm{C}-4), 171.7(\mathrm{C}=\mathrm{O})$; LCMS $(m / z)$ : $453\left(\mathrm{M}^{+}\right)$; Anal. Calcd. For $\mathrm{C}_{21} \mathrm{H}_{13} \mathrm{Cl}_{2} \mathrm{~N}_{5} \mathrm{O}_{3}$ : C-55.52, H-2.88, N-15.42; Found: C-55.44, H-2.80, N-15.47\%.

2.41 2-Chloro-N-(4-(2-chloroquinolin-3-yl)-6-(4nitrophenyl)pyrimidin-2-yl)acetamide (4l): Dark yellow crystal, yield: $73 \%$; m.p.: 224-226 ${ }^{\circ} \mathrm{C}$; IR (KBr) $v_{\max } / \mathrm{cm}^{-1}: 3369,3283(\mathrm{NH}), 3088(\mathrm{C}-\mathrm{H}$, aromatic), $2862\left(\mathrm{C}-\mathrm{H}, \mathrm{CH}_{2}\right), 1686(\mathrm{C}=\mathrm{O}), 1582(\mathrm{C}=\mathrm{N}), 1522$ $(\mathrm{C}=\mathrm{C}), 1478,1350\left(\mathrm{NO}_{2}\right), 790(\mathrm{C}-\mathrm{Cl}) ;{ }^{1} \mathrm{H}$ NMR $\left(300 \mathrm{MHz}, \mathrm{DMSO}-d_{6}, \delta, \mathrm{ppm}\right): 4.29\left(\mathrm{~s}, 2 \mathrm{H}, \mathrm{CH}_{2}\right)$, $6.95(\mathrm{~s}, 1 \mathrm{H}$, pyrimidine-H), 7.56-8.58 (m, 9H, Ar-H), 9.79 (s, $1 \mathrm{H}, \mathrm{NH}, \mathrm{D}_{2} \mathrm{O}$ exch.); ${ }^{13} \mathrm{C}$ NMR $(100 \mathrm{MHz}$, DMSO- $\left.d_{6}, \delta, \mathrm{ppm}\right): 60.5\left(\mathrm{CH}_{2}\right), 101.3(\mathrm{C}-5), 124.1-$ 147.4 (C-arom), 152.4 (C-Cl, quinoline), 162.2 (C-2), 164.5 (C-6), $165.8(\mathrm{C}-4), 171.4(\mathrm{C}=\mathrm{O})$; LCMS $(\mathrm{m} / \mathrm{z})$ : $453\left(\mathrm{M}^{+}\right)$; Anal. Calcd. For $\mathrm{C}_{21} \mathrm{H}_{13} \mathrm{Cl}_{2} \mathrm{~N}_{5} \mathrm{O}_{3}$ : C-55.52, H-2.88, N-15.42; Found: C-55.48, H-2.82, N-15.37\%.

2.5 General procedure for the preparation of $\mathrm{N}-(4-$ (2-chloroquinolin-3-yl)-6-(aryl)pyrimidin-2-yl)-2morpholinoacetamides (5a-l)

A mixture of compounds (4a-l) $(0.01 \mathrm{~mol})$, anhydrous potassium carbonate $(0.02 \mathrm{~mol})$ and morpholine $(0.01 \mathrm{~mol})$ in dry toluene was refluxed for about 8 $10 \mathrm{~h}$. After completion of the reaction, potassium carbonate was removed by filtration and excess of solvent was removed under reduced pressure. The obtained residues were filtered, dried and crystallized from ethanol $(95 \%)$.

2.5a N-(4-(2-chloroquinolin-3-yl)-6-phenylpyrimidin2-yl)-2-morpholinoacetamide (5a): Light brown crystal, yield: $57 \%$; m.p.: $204-206^{\circ} \mathrm{C}$; IR (KBr) $v_{\max } / \mathrm{cm}^{-1}$ : 3314, $3230(\mathrm{NH}), 3071$ (C-H, aromatic), 2962, 2920, $2850\left(\mathrm{C}-\mathrm{H}, \mathrm{CH}_{2}\right), 1686(\mathrm{C}=\mathrm{O}), 1605(\mathrm{C}=\mathrm{N}), 1570$ $(\mathrm{C}=\mathrm{C}), 1360(\mathrm{C}-\mathrm{N}), 1236(\mathrm{C}-\mathrm{O}), 720(\mathrm{C}-\mathrm{Cl}) ;{ }^{1} \mathrm{H}$ NMR (300 MHz, DMSO- $\left.d_{6}, \delta, \mathrm{ppm}\right): 2.61(\mathrm{t}, 4 \mathrm{H}, J=4.5 \mathrm{~Hz}$, $\left.\mathrm{N}\left(\mathrm{CH}_{2}\right)_{2}\right), 3.47\left(\mathrm{t}, 4 \mathrm{H}, J=4.8 \mathrm{~Hz}, \mathrm{O}\left(\mathrm{CH}_{2}\right)_{2}\right), 3.91$ (s, 2H, $\left.\mathrm{CH}_{2}\right), 6.73(\mathrm{~s}, 1 \mathrm{H}$, pyrimidine-H), 7.34-8.47 (m, 10H, Ar-H), 10.21 (s, $1 \mathrm{H}, \mathrm{NH}, \mathrm{D}_{2} \mathrm{O}$ exch.); ${ }^{13} \mathrm{C}$
NMR (100 MHz, DMSO- $\left.d_{6}, \delta, \mathrm{ppm}\right): 45.3\left(\mathrm{~N}\left(\mathrm{CH}_{2}\right)_{2}\right)$, $63.2\left(\mathrm{CH}_{2}\right), 67.3\left(\mathrm{O}\left(\mathrm{CH}_{2}\right)_{2}\right), 101.1(\mathrm{C}-5), 126.4-145.2$ (C-arom), 151.8 (C-Cl, quinoline), 163.4 (C-2), 164.5 (C-6), 165.3 (C-4), $173.1 \quad(\mathrm{C}=\mathrm{O})$; LCMS $(\mathrm{m} / \mathrm{z})$ : $459\left(\mathrm{M}^{+}\right)$; Anal. Calcd. For $\mathrm{C}_{25} \mathrm{H}_{22} \mathrm{ClN}_{5} \mathrm{O}_{2}$ : C-65.29, H-4.82, N-15.23; Found: C-65.34, H-4.87, N-15.16\%.

2.5b N-(4-(2-chloroquinolin-3-yl)-6-(2-hydroxyphenyl) pyrimidin-2-yl)-2-morpholinoacetamide (5b): Light yellow crystal, yield: 53\%; m.p.: $164-166^{\circ} \mathrm{C}$; IR (KBr) $v_{\max } / \mathrm{cm}^{-1}$ : $3412(\mathrm{OH}), 3310,3236(\mathrm{NH}), 3068(\mathrm{C}-\mathrm{H}$, aromatic), 2964, 2929, $2854\left(\mathrm{C}-\mathrm{H}, \mathrm{CH}_{2}\right), 1682(\mathrm{C}=\mathrm{O})$, $1589(\mathrm{C}=\mathrm{N}), 1575(\mathrm{C}=\mathrm{C}), 1357(\mathrm{C}-\mathrm{N}), 1230(\mathrm{C}-\mathrm{O})$, $726(\mathrm{C}-\mathrm{Cl}) ;{ }^{1} \mathrm{H}$ NMR $\left(300 \mathrm{MHz}, \mathrm{DMSO}-d_{6}, \delta, \mathrm{ppm}\right)$ : $2.65\left(\mathrm{t}, 4 \mathrm{H}, J=4.9 \mathrm{~Hz}, \mathrm{~N}\left(\mathrm{CH}_{2}\right)_{2}\right), 3.45(\mathrm{t}, 4 \mathrm{H}, J=$ $\left.4.7 \mathrm{~Hz}, \mathrm{O}\left(\mathrm{CH}_{2}\right)_{2}\right), 3.94\left(\mathrm{~s}, 2 \mathrm{H}, \mathrm{CH}_{2}\right), 6.78(\mathrm{~s}, 1 \mathrm{H}$, pyrimidine- $\mathrm{H}), 7.01-8.42(\mathrm{~m}, 9 \mathrm{H}, \mathrm{Ar}-\mathrm{H}), 9.21(\mathrm{~s}, 1 \mathrm{H}$, $\mathrm{OH}, \mathrm{D}_{2} \mathrm{O}$ exch.), 10.18 (s, $1 \mathrm{H}, \mathrm{NH}, \mathrm{D}_{2} \mathrm{O}$ exch.); ${ }^{13} \mathrm{C}$ NMR (100 MHz, DMSO- $\left.d_{6}, \delta, \mathrm{ppm}\right): 45.5\left(\mathrm{~N}\left(\mathrm{CH}_{2}\right)_{2}\right)$, $63.8\left(\mathrm{CH}_{2}\right), 67.3\left(\mathrm{O}\left(\mathrm{CH}_{2}\right)_{2}\right), 101.7(\mathrm{C}-5), 117.6-154.4$ (C-arom), 151.6 (C-Cl, quinoline), 163.2 (C-2), 164.3 (C-6), $165.5(\mathrm{C}-4), 173.3(\mathrm{C}=\mathrm{O})$; LCMS $(\mathrm{m} / \mathrm{z})$ : $475\left(\mathrm{M}^{+}\right)$; Anal. Calcd. For $\mathrm{C}_{25} \mathrm{H}_{22} \mathrm{ClN}_{5} \mathrm{O}_{3}$ : C-63.09, H-4.66, N-14.72; Found: C-63.14, H-4.73, N-14.65\%.

2.5c N-(4-(2-chloroquinolin-3-yl)-6-(4-hydroxyphenyl) pyrimidin-2-yl)-2-morpholinoacetamide (5c): Light reddish brown crystal, yield: $51 \%$; m.p.: $205-206^{\circ} \mathrm{C}$; IR $(\mathrm{KBr}) v_{\max } / \mathrm{cm}^{-1}: 3419(\mathrm{OH}), 3316,3226(\mathrm{NH})$, 3062 (C-H, aromatic), 2960, 2931, $2850\left(\mathrm{C}-\mathrm{H}, \mathrm{CH}_{2}\right)$, $1690(\mathrm{C}=\mathrm{O}), 1583(\mathrm{C}=\mathrm{N}), 1579(\mathrm{C}=\mathrm{C}), 1357(\mathrm{C}-\mathrm{N})$, $1222(\mathrm{C}-\mathrm{O}), 731(\mathrm{C}-\mathrm{Cl})$; ${ }^{1} \mathrm{H}$ NMR $(300 \mathrm{MHz}$, DMSO$\left.d_{6}, \delta, \mathrm{ppm}\right): 2.68\left(\mathrm{t}, 4 \mathrm{H}, J=4.4 \mathrm{~Hz}, \mathrm{~N}\left(\mathrm{CH}_{2}\right)_{2}\right), 3.42(\mathrm{t}$, $\left.4 \mathrm{H}, J=4.6 \mathrm{~Hz}, \mathrm{O}\left(\mathrm{CH}_{2}\right)_{2}\right), 3.90\left(\mathrm{~s}, 2 \mathrm{H}, \mathrm{CH}_{2}\right), 6.71(\mathrm{~s}$, $1 \mathrm{H}$, pyrimidine-H), 6.88-8.44 (m, 9H, Ar-H), 9.17 (s, 1H, OH, $\mathrm{D}_{2} \mathrm{O}$ exch.), 10.25 (s, $1 \mathrm{H}, \mathrm{NH}, \mathrm{D}_{2} \mathrm{O}$ exch.); ${ }^{13} \mathrm{C}$ NMR (100 MHz, DMSO- $\left.d_{6}, \delta, \mathrm{ppm}\right): 45.7\left(\mathrm{~N}\left(\mathrm{CH}_{2}\right)_{2}\right)$, $63.5\left(\mathrm{CH}_{2}\right), 67.7\left(\mathrm{O}\left(\mathrm{CH}_{2}\right)_{2}\right), 101.5(\mathrm{C}-5), 115.7-156.8$ (C-arom), 151.6 (C-Cl, quinoline), $163.2(\mathrm{C}-2), 164.4$ (C-6), 165.3 (C-4), $173.8(\mathrm{C}=\mathrm{O})$; LCMS $(\mathrm{m} / \mathrm{z})$ : $475\left(\mathrm{M}^{+}\right)$; Anal. Calcd. For $\mathrm{C}_{25} \mathrm{H}_{22} \mathrm{ClN}_{5} \mathrm{O}_{3}$ : C-63.09, H-4.66, N-14.72; Found: C-63.17, H-4.60, N-14.74\%.

2.5d N-(4-(2-chloroquinolin-3-yl)-6-(4-methoxyphenyl) pyrimidin-2-yl)-2-morpholinoacetamide (5d): Light pink crystal, yield: $55 \%$; m.p.: $171-173^{\circ} \mathrm{C}$; IR (KBr) $v_{\max } / \mathrm{cm}^{-1}$ : 3320, $3215(\mathrm{NH}), 3074(\mathrm{C}-\mathrm{H}$, aromatic), 2966, 2938, $2857\left(\mathrm{C}-\mathrm{H}, \mathrm{CH}_{2}\right), 1688(\mathrm{C}=\mathrm{O}), 1590$ $(\mathrm{C}=\mathrm{N}), 1574(\mathrm{C}=\mathrm{C}), 1351(\mathrm{C}-\mathrm{N}), 1231,1134(\mathrm{C}-$ O), $729(\mathrm{C}-\mathrm{Cl}) ;{ }^{1} \mathrm{H}$ NMR $\left(300 \mathrm{MHz}, \mathrm{DMSO}-d_{6}, \delta\right.$, ppm): 2.63 (t, $\left.4 \mathrm{H}, J=4.7 \mathrm{~Hz}, \mathrm{~N}\left(\mathrm{CH}_{2}\right)_{2}\right), 3.49$ (t, $4 \mathrm{H}$, 
$\left.J=4.5 \mathrm{~Hz}, \mathrm{O}\left(\mathrm{CH}_{2}\right)_{2}\right), 3.64\left(\mathrm{~s}, 3 \mathrm{H}, \mathrm{OCH}_{3}\right), 3.96(\mathrm{~s}$, $\left.2 \mathrm{H}, \mathrm{CH}_{2}\right), 6.82(\mathrm{~s}, 1 \mathrm{H}$, pyrimidine- $\mathrm{H}), 7.09-8.44(\mathrm{~m}$, 9H, Ar-H), 10.10 (s, 1H, NH, $\mathrm{D}_{2} \mathrm{O}$ exch.); ${ }^{13} \mathrm{C}$ NMR $\left(100 \mathrm{MHz}, \mathrm{DMSO}-d_{6}, \delta, \mathrm{ppm}\right): 45.5\left(\mathrm{~N}\left(\mathrm{CH}_{2}\right)_{2}\right), 55.4$ $\left(\mathrm{OCH}_{3}\right), 63.6\left(\mathrm{CH}_{2}\right), 67.2\left(\mathrm{O}\left(\mathrm{CH}_{2}\right)_{2}\right), 101.5(\mathrm{C}-5)$, 113.8-159.3 (C-arom), 152.3 (C-Cl, quinoline), 163.7 (C-2), 164.4 (C-6), 165.7 (C-4), $173.4(\mathrm{C}=\mathrm{O})$; LCMS $(\mathrm{m} / z)$ : $489\left(\mathrm{M}^{+}\right)$; Anal. Calcd. For $\mathrm{C}_{26} \mathrm{H}_{24} \mathrm{ClN}_{5} \mathrm{O}_{3}$ : C-63.74, H-4.94, N-14.29; Found: C-63.81, H-4.91, $\mathrm{N}-22 \%$.

2.5e N-(4-(2-chlorophenyl)-6-(2-chloroquinolin-3-yl) pyrimidin-2-yl)-2-morpholinoacetamide (5e): Light yellow crystal, yield: $58 \%$; m.p.: $182-184^{\circ} \mathrm{C}$; IR ( $\left.\mathrm{KBr}\right)$ $v_{\max } / \mathrm{cm}^{-1}: 3320,3214(\mathrm{NH}), 3062(\mathrm{C}-\mathrm{H}$, aromatic), 2968, 2933, $2858\left(\mathrm{C}-\mathrm{H}, \mathrm{CH}_{2}\right), 1698(\mathrm{C}=\mathrm{O}), 1601$ $(\mathrm{C}=\mathrm{N}), 1565(\mathrm{C}=\mathrm{C}), 1352(\mathrm{C}-\mathrm{N}), 1238(\mathrm{C}-\mathrm{O}), 739$ $(\mathrm{C}-\mathrm{Cl}) ;{ }^{1} \mathrm{H}$ NMR $\left(300 \mathrm{MHz}, \mathrm{DMSO}-d_{6}, \delta, \mathrm{ppm}\right): 2.69$ $\left(\mathrm{t}, 4 \mathrm{H}, J=4.3 \mathrm{~Hz}, \mathrm{~N}\left(\mathrm{CH}_{2}\right)_{2}\right), 3.49(\mathrm{t}, 4 \mathrm{H}, J=4.9 \mathrm{~Hz}$, $\left.\mathrm{O}\left(\mathrm{CH}_{2}\right)_{2}\right), 3.92\left(\mathrm{~s}, 2 \mathrm{H}, \mathrm{CH}_{2}\right), 6.79(\mathrm{~s}, 1 \mathrm{H}$, pyrimidine$\mathrm{H}), 7.33-8.49(\mathrm{~m}, 9 \mathrm{H}, \mathrm{Ar}-\mathrm{H}), 10.27\left(\mathrm{~s}, 1 \mathrm{H}, \mathrm{NH}, \mathrm{D}_{2} \mathrm{O}\right.$ exch.); ${ }^{13} \mathrm{C}$ NMR (100 MHz, DMSO- $\left.d_{6}, \delta, \mathrm{ppm}\right): 45.1$ $\left(\mathrm{N}\left(\mathrm{CH}_{2}\right)_{2}\right), 63.3\left(\mathrm{CH}_{2}\right), 67.7\left(\mathrm{O}\left(\mathrm{CH}_{2}\right)_{2}\right), 101.3(\mathrm{C}-5)$, 126.2-146.4 (C-arom), 152.5 (C-Cl, quinoline), 163.4 (C-2), 164.8 (C-6), 165.1 (C-4), $173.5(\mathrm{C}=\mathrm{O})$; LCMS $(m / z)$ : $493\left(\mathrm{M}^{+}\right)$; Anal. Calcd. For $\mathrm{C}_{25} \mathrm{H}_{21} \mathrm{Cl}_{2} \mathrm{~N}_{5} \mathrm{O}_{2}$ : C-60.74, H-4.28, N-14.17; Found: C-60.69, H-4.32, $\mathrm{N}-14.10 \%$.

$2.5 \mathrm{f} \quad \mathrm{N}$-(4-(4-chlorophenyl)-6-(2-chloroquinolin-3-yl) pyrimidin-2-yl)-2-morpholinoacetamide (5f): Light reddish brown crystal, yield: $50 \%$; m.p.: $167-169^{\circ} \mathrm{C}$; IR $(\mathrm{KBr}) v_{\max } / \mathrm{cm}^{-1}: 3332,3219(\mathrm{NH}), 3067(\mathrm{C}-\mathrm{H}$, aromatic), 2961, 2928, $2858\left(\mathrm{C}-\mathrm{H}, \mathrm{CH}_{2}\right), 1690(\mathrm{C}=\mathrm{O})$, $1594(\mathrm{C}=\mathrm{N}), 1560(\mathrm{C}=\mathrm{C}), 1361(\mathrm{C}-\mathrm{N}), 1238(\mathrm{C}-\mathrm{O})$, $732(\mathrm{C}-\mathrm{Cl}) ;{ }^{1} \mathrm{H}$ NMR $\left(300 \mathrm{MHz}, \mathrm{DMSO}-d_{6}, \delta, \mathrm{ppm}\right)$ : $2.66\left(\mathrm{t}, 4 \mathrm{H}, J=4.5 \mathrm{~Hz}, \mathrm{~N}\left(\mathrm{CH}_{2}\right)_{2}\right), 3.44(\mathrm{t}, 4 \mathrm{H}, J=$ $\left.4.3 \mathrm{~Hz}, \mathrm{O}\left(\mathrm{CH}_{2}\right)_{2}\right), 3.94\left(\mathrm{~s}, 2 \mathrm{H}, \mathrm{CH}_{2}\right), 6.74(\mathrm{~s}, 1 \mathrm{H}$, pyrimidine-H), 7.50-8.54 (m, 9H, Ar-H), 10.24 (s, 1H, $\mathrm{NH}, \mathrm{D}_{2} \mathrm{O}$ exch.); ${ }^{13} \mathrm{C}$ NMR $\left(100 \mathrm{MHz}, \mathrm{DMSO}-d_{6}, \delta\right.$,

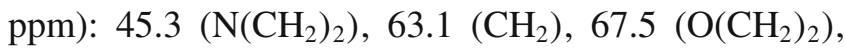
101.6 (C-5), 127.5-145.3 (C-arom), 152.2 (C-Cl, quinoline), 163.1 (C-2), 164.4 (C-6), 165.2 (C-4), $173.4(\mathrm{C}=\mathrm{O})$; LCMS $(\mathrm{m} / \mathrm{z}): 493\left(\mathrm{M}^{+}\right)$; Anal. Calcd. For $\mathrm{C}_{25} \mathrm{H}_{21} \mathrm{Cl}_{2} \mathrm{~N}_{5} \mathrm{O}_{2}$ : C-60.74, H-4.28, N-14.17; Found: C-60.82, H-4.35, N-14.26\%.

$2.5 \mathrm{~g} \quad \mathrm{~N}$-(4-(2-chloroquinolin-3-yl)-6-(2-fluorophenyl) pyrimidin-2-yl)-2-morpholinoacetamide (5g): Light yellow crystal, yield: $56 \%$; m.p.: $188-190^{\circ} \mathrm{C}$; IR (KBr) $v_{\max } / \mathrm{cm}^{-1}$ : 3326, $3228(\mathrm{NH}), 3071(\mathrm{C}-\mathrm{H}$, aromatic),
2958, 2920, $2854\left(\mathrm{C}-\mathrm{H}, \mathrm{CH}_{2}\right), 1688(\mathrm{C}=\mathrm{O}), 1588$ $(\mathrm{C}=\mathrm{N}), 1562(\mathrm{C}=\mathrm{C}), 1355(\mathrm{C}-\mathrm{N}), 1230(\mathrm{C}-\mathrm{O}), 1117$ (C-F), $741(\mathrm{C}-\mathrm{Cl}) ;{ }^{1} \mathrm{H}$ NMR $\left(300 \mathrm{MHz}, \mathrm{DMSO}-d_{6}\right.$, $\delta, \mathrm{ppm}): 2.71\left(\mathrm{t}, 4 \mathrm{H}, J=4.7 \mathrm{~Hz}, \mathrm{~N}\left(\mathrm{CH}_{2}\right)_{2}\right), 3.52$ $\left(\mathrm{t}, 4 \mathrm{H}, J=4.1 \mathrm{~Hz}, \mathrm{O}\left(\mathrm{CH}_{2}\right)_{2}\right), 3.93\left(\mathrm{~s}, 2 \mathrm{H}, \mathrm{CH}_{2}\right)$, $6.84(\mathrm{~s}, 1 \mathrm{H}$, pyrimidine-H), 7.17-8.58 (m, 9H, Ar-H), 10.27 (s, $1 \mathrm{H}, \mathrm{NH}, \mathrm{D}_{2} \mathrm{O}$ exch.); ${ }^{13} \mathrm{C}$ NMR $(100 \mathrm{MHz}$, DMSO- $\left.d_{6}, \delta, \mathrm{ppm}\right): 45.5\left(\mathrm{~N}\left(\mathrm{CH}_{2}\right)_{2}\right), 63.7\left(\mathrm{CH}_{2}\right), 67.2$ $\left(\mathrm{O}\left(\mathrm{CH}_{2}\right)_{2}\right), 101.5(\mathrm{C}-5), 114.3-158.2$ (C-arom), 153.1 (C-Cl, quinoline), 163.2 (C-2), 164.5 (C-6), 165.6 $(\mathrm{C}-4), 173.2(\mathrm{C}=\mathrm{O})$; LCMS $(\mathrm{m} / z): 477\left(\mathrm{M}^{+}\right)$; Anal. Calcd. For $\mathrm{C}_{25} \mathrm{H}_{21} \mathrm{ClFN}_{5} \mathrm{O}_{2}$ : C-62.83, H-4.43, N-14.65; Found: C-62.88, H-4.38, N-14.61\%.

2.5h N-(4-(2-chloroquinolin-3-yl)-6-(3-fluorophenyl) pyrimidin-2-yl)-2-morpholinoacetamide (5h): Light pink crystal, yield: $54 \%$; m.p.: $177-179^{\circ} \mathrm{C}$; IR (KBr) $v_{\max } / \mathrm{cm}^{-1}: 3339,3241(\mathrm{NH}), 3078(\mathrm{C}-\mathrm{H}$, aromatic), 2961, 2914, $2844\left(\mathrm{C}-\mathrm{H}, \mathrm{CH}_{2}\right), 1687(\mathrm{C}=\mathrm{O}), 1580$ $(\mathrm{C}=\mathrm{N}), 1559(\mathrm{C}=\mathrm{C}), 1348(\mathrm{C}-\mathrm{N}), 1239(\mathrm{C}-\mathrm{O}), 1120$ (C-F), 749 (C-Cl); ${ }^{1} \mathrm{H}$ NMR $\left(300 \mathrm{MHz}\right.$, DMSO- $d_{6}$, $\delta, \mathrm{ppm}): 2.75\left(\mathrm{t}, 4 \mathrm{H}, J=4.8 \mathrm{~Hz}, \mathrm{~N}\left(\mathrm{CH}_{2}\right)_{2}\right), 3.58$ $\left(\mathrm{t}, 4 \mathrm{H}, J=4.6 \mathrm{~Hz}, \mathrm{O}\left(\mathrm{CH}_{2}\right)_{2}\right), 3.96\left(\mathrm{~s}, 2 \mathrm{H}, \mathrm{CH}_{2}\right)$, $6.80(\mathrm{~s}, 1 \mathrm{H}$, pyrimidine-H), 7.25-8.55 (m, 9H, Ar-H), 10.31 (s, $1 \mathrm{H}, \mathrm{NH}, \mathrm{D}_{2} \mathrm{O}$ exch.); ${ }^{13} \mathrm{C}$ NMR $(100 \mathrm{MHz}$, DMSO- $\left.d_{6}, \delta, \mathrm{ppm}\right): 45.7\left(\mathrm{~N}\left(\mathrm{CH}_{2}\right)_{2}\right), 63.8\left(\mathrm{CH}_{2}\right), 67.1$ $\left(\mathrm{O}\left(\mathrm{CH}_{2}\right)_{2}\right), 101.0$ (C-5), 115.1-161.7 (C-arom), 153.3 (C-Cl, quinoline), 163.1 (C-2), 164.4 (C-6), 165.3 (C-4), $173.0(\mathrm{C}=\mathrm{O})$; LCMS $(\mathrm{m} / \mathrm{z})$ : $477\left(\mathrm{M}^{+}\right)$; Anal. Calcd. For $\mathrm{C}_{25} \mathrm{H}_{21} \mathrm{ClFN}_{5} \mathrm{O}_{2}$ : C-62.83, H-4.43, N-14.65; Found: C-62.77, H-4.47, N-14.72\%.

$2.5 \mathrm{i} \quad \mathrm{N}$-(4-(2-chloroquinolin-3-yl)-6-(4-fluorophenyl) pyrimidin-2-yl)-2-morpholinoacetamide (5i): Light brown crystal, yield: 59\%; m.p.: $211-213^{\circ} \mathrm{C}$; IR (KBr) $v_{\max } / \mathrm{cm}^{-1}: 3359,3240(\mathrm{NH}), 3064(\mathrm{C}-\mathrm{H}$, aromatic), 2973, 2951, $2840\left(\mathrm{C}-\mathrm{H}, \mathrm{CH}_{2}\right), 1691(\mathrm{C}=\mathrm{O}), 1576$ $(\mathrm{C}=\mathrm{N}), 1532(\mathrm{C}=\mathrm{C}), 1359(\mathrm{C}-\mathrm{N}), 1231(\mathrm{C}-\mathrm{O}), 1138$ (C-F), $737(\mathrm{C}-\mathrm{Cl}) ;{ }^{1} \mathrm{H}$ NMR $\left(300 \mathrm{MHz}, \mathrm{DMSO}-d_{6}\right.$, $\delta, \mathrm{ppm}): 2.73\left(\mathrm{t}, 4 \mathrm{H}, J=4.7 \mathrm{~Hz}, \mathrm{~N}\left(\mathrm{CH}_{2}\right)_{2}\right), 3.55$ $\left(\mathrm{t}, 4 \mathrm{H}, J=4.4 \mathrm{~Hz}, \mathrm{O}\left(\mathrm{CH}_{2}\right)_{2}\right), 3.91\left(\mathrm{~s}, 2 \mathrm{H}, \mathrm{CH}_{2}\right)$, 6.77 (s, 1H, pyrimidine-H), 7.30-8.52 (m, 9H, Ar-H), 10.29 (s, $1 \mathrm{H}, \mathrm{NH}, \mathrm{D}_{2} \mathrm{O}$ exch.); ${ }^{13} \mathrm{C}$ NMR $(100 \mathrm{MHz}$, DMSO- $\left.d_{6}, \delta, \mathrm{ppm}\right): 45.2\left(\mathrm{~N}\left(\mathrm{CH}_{2}\right)_{2}\right), 63.6\left(\mathrm{CH}_{2}\right), 67.7$ $\left(\mathrm{O}\left(\mathrm{CH}_{2}\right)_{2}\right), 101.3(\mathrm{C}-5), 115.7-162.3$ (C-arom), 152.8 (C-Cl, quinoline), 163.5 (C-2), 164.2 (C-6), 165.7 $(\mathrm{C}-4), 173.3(\mathrm{C}=\mathrm{O})$; LCMS $(\mathrm{m} / \mathrm{z})$ : $477\left(\mathrm{M}^{+}\right)$; Anal. Calcd. For $\mathrm{C}_{25} \mathrm{H}_{21} \mathrm{ClFN}_{5} \mathrm{O}_{2}$ : C-62.83, H-4.43, N-14.65; Found: C-62.87, H-4.38, N-14.70\%. 
2.5j N-(4-(2-chloroquinolin-3-yl)-6-(2-nitrophenyl) pyrimidin-2-yl)-2-morpholinoacetamide (5j): Yellow orange crystal, yield: $73 \%$; m.p.: $224-226^{\circ} \mathrm{C}$; IR (KBr) $v_{\max } / \mathrm{cm}^{-1}: 3350,3234(\mathrm{NH}), 3071(\mathrm{C}-\mathrm{H}$, aromatic), 2983, 2956, $2831\left(\mathrm{C}-\mathrm{H}, \mathrm{CH}_{2}\right), 1697(\mathrm{C}=\mathrm{O}), 1593$ $(\mathrm{C}=\mathrm{N}), 1538(\mathrm{C}=\mathrm{C}), 1485,1347\left(\mathrm{NO}_{2}\right.$ group $), 1363$ (C-N), 1233 (C-O), 730 (C-Cl); ${ }^{1} \mathrm{H}$ NMR $(300 \mathrm{MHz}$, DMSO- $\left.d_{6}, \delta, \mathrm{ppm}\right): 2.76\left(\mathrm{t}, 4 \mathrm{H}, J=4.9 \mathrm{~Hz}, \mathrm{~N}\left(\mathrm{CH}_{2}\right)_{2}\right)$, $3.51\left(\mathrm{t}, 4 \mathrm{H}, J=4.7 \mathrm{~Hz}, \mathrm{O}\left(\mathrm{CH}_{2}\right)_{2}\right), 3.95\left(\mathrm{~s}, 2 \mathrm{H}, \mathrm{CH}_{2}\right)$, $6.82(\mathrm{~s}, 1 \mathrm{H}$, pyrimidine-H), 7.62-8.56 (m, 9H, Ar-H), 10.34 (s, 1H, NH, $\mathrm{D}_{2} \mathrm{O}$ exch.); ${ }^{13} \mathrm{C}$ NMR $(100 \mathrm{MHz}$, DMSO- $\left.d_{6}, \delta, \mathrm{ppm}\right): 45.4\left(\mathrm{~N}\left(\mathrm{CH}_{2}\right)_{2}\right), 63.7\left(\mathrm{CH}_{2}\right), 67.9$ $\left(\mathrm{O}\left(\mathrm{CH}_{2}\right)_{2}\right), 101.3$ (C-5), 124.2-148.4 (C-arom), 152.6 (C-Cl, quinoline), 163.7 (C-2), 164.2 (C-6), 165.3 (C-4), $173.7(\mathrm{C}=\mathrm{O})$; LCMS $(\mathrm{m} / \mathrm{z})$ : $504\left(\mathrm{M}^{+}\right)$; Anal. Calcd. For $\mathrm{C}_{25} \mathrm{H}_{21} \mathrm{ClN}_{6} \mathrm{O}_{4}$ : C-59.47, H-4.19, N-16.64; Found: C-59.41, H-4.14, N-16.70\%.

2.5k N-(4-(2-chloroquinolin-3-yl)-6-(3-nitrophenyl) pyrimidin-2-yl)-2-morpholinoacetamide (5k): Dark yellow crystal, yield: $52 \%$; m.p.: $155-157^{\circ} \mathrm{C}$; IR (KBr) $v_{\max } / \mathrm{cm}^{-1}: 3369,3258(\mathrm{NH}), 3065(\mathrm{C}-\mathrm{H}$, aromatic), 2980, 2947, $2835\left(\mathrm{C}-\mathrm{H}, \mathrm{CH}_{2}\right), 1690(\mathrm{C}=\mathrm{O}), 1584$ $(\mathrm{C}=\mathrm{N}), 1522(\mathrm{C}=\mathrm{C}), 1482,1348\left(\mathrm{NO}_{2}\right.$ group $), 1360$ (C-N), 1235 (C-O), 725 (C-Cl); ${ }^{1} \mathrm{H}$ NMR $(300 \mathrm{MHz}$, DMSO- $\left.d_{6}, \delta, \mathrm{ppm}\right): 2.74$ (t, $\left.4 \mathrm{H}, J=4.6 \mathrm{~Hz}, \mathrm{~N}\left(\mathrm{CH}_{2}\right)_{2}\right)$, $3.56\left(\mathrm{t}, 4 \mathrm{H}, J=4.3 \mathrm{~Hz}, \mathrm{O}\left(\mathrm{CH}_{2}\right)_{2}\right), 3.92\left(\mathrm{~s}, 2 \mathrm{H}, \mathrm{CH}_{2}\right)$, $6.80(\mathrm{~s}, 1 \mathrm{H}$, pyrimidine-H), 7.70-8.67 (m, 9H, Ar-H), 10.30 (s, $1 \mathrm{H}, \mathrm{NH}, \mathrm{D}_{2} \mathrm{O}$ exch.); ${ }^{13} \mathrm{C}$ NMR $(100 \mathrm{MHz}$, DMSO- $\left.d_{6}, \delta, \mathrm{ppm}\right): 45.1\left(\mathrm{~N}\left(\mathrm{CH}_{2}\right)_{2}\right), 63.4\left(\mathrm{CH}_{2}\right), 67.7$ $\left(\mathrm{O}\left(\mathrm{CH}_{2}\right)_{2}\right), 101.5$ (C-5), 122.7-147.8 (C-arom), 152.7 (C-Cl, quinoline), 163.2 (C-2), 164.6 (C-6), 165.8 (C-4), $173.6(\mathrm{C}=\mathrm{O})$; LCMS $(m / z): 504\left(\mathrm{M}^{+}\right)$; Anal. Calcd. For $\mathrm{C}_{25} \mathrm{H}_{21} \mathrm{ClN}_{6} \mathrm{O}_{4}$ : C-59.47, H-4.19, N-16.64; Found: C-59.52, H-4.25, N-16.59\%.

\section{$2.51 \quad \mathrm{~N}$-(4-(2-chloroquinolin-3-yl)-6-(4-nitrophenyl)} pyrimidin-2-yl)-2-morpholinoacetamide (5l): Dark orange yellow crystal, yield: $55 \%$; m.p.: $188-190^{\circ} \mathrm{C}$; IR $(\mathrm{KBr}) v_{\max } / \mathrm{cm}^{-1}$ : 3353, $3244(\mathrm{NH}), 3079(\mathrm{C}-\mathrm{H}$, aromatic), 2973, 2940, $2828\left(\mathrm{C}-\mathrm{H}, \mathrm{CH}_{2}\right), 1698(\mathrm{C}=\mathrm{O})$, $1594(\mathrm{C}=\mathrm{N}), 1515(\mathrm{C}=\mathrm{C}), 1475,1355\left(\mathrm{NO}_{2}\right.$ group $)$, $1363(\mathrm{C}-\mathrm{N}), 1241$ (C-O), $732(\mathrm{C}-\mathrm{Cl}) ;{ }^{1} \mathrm{H}$ NMR (300 MHz, DMSO- $\left.d_{6}, \delta, \mathrm{ppm}\right): 2.70(\mathrm{t}, 4 \mathrm{H}, J=4.5 \mathrm{~Hz}$, $\left.\mathrm{N}\left(\mathrm{CH}_{2}\right)_{2}\right), 3.54\left(\mathrm{t}, 4 \mathrm{H}, J=4.8 \mathrm{~Hz}, \mathrm{O}\left(\mathrm{CH}_{2}\right)_{2}\right), 3.95$ (s, $\left.2 \mathrm{H}, \mathrm{CH}_{2}\right), 6.75(\mathrm{~s}, 1 \mathrm{H}$, pyrimidine-H), 7.63-8.58 (m, 9H, Ar-H), 10.36 (s, 1H, NH, $\mathrm{D}_{2} \mathrm{O}$ exch.); ${ }^{13} \mathrm{C}$

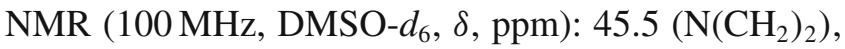
$63.2\left(\mathrm{CH}_{2}\right), 67.5\left(\mathrm{O}\left(\mathrm{CH}_{2}\right)_{2}\right), 101.2(\mathrm{C}-5), 124.3-146.8$ (C-arom), 151.8 (C-Cl, quinoline), 163.8 (C-2), 164.5 (C-6), 165.2 (C-4), $173.7(\mathrm{C}=\mathrm{O})$; LCMS $(m / z)$ :
$504\left(\mathrm{M}^{+}\right)$; Anal. Calcd. For $\mathrm{C}_{25} \mathrm{H}_{21} \mathrm{ClN}_{6} \mathrm{O}_{4}$ : C-59.47, H-4.19, N-16.64; Found: C-59.55, H-4.25, N-16.72\%.

\section{Materials and methods}

\subsection{General}

All reactions except those in aqueous media were carried out by standard techniques for the exclusion of moisture. Melting points were determined on an electro thermal melting point apparatus and were reported uncorrected. TLC on silica gel plates (Merck, 60, $\mathrm{F}_{254}$ ) was used for purity check and reaction monitoring. Column chromatography on silica gel (Merck, 70-230 mesh and 230-400 mesh ASTH for flash chromatography) was applied when necessary to isolate and purify the reactions products. Elemental analysis $(\% \mathrm{C}, \mathrm{H}, \mathrm{N})$ was carried out by a Perkin-Elmer $2400 \mathrm{CHN}$ analyser. IR spectra of all compounds have been recorded on a Perkin-Elmer FT-IR spectrometer in $\mathrm{KBr} .{ }^{1} \mathrm{H}$ NMR spectra were recorded on Varian Gemini $300 \mathrm{MHz}$ and ${ }^{13} \mathrm{C}$ NMR spectra on Varian Mercury-400, $100 \mathrm{MHz}$ in DMSO- $d_{6}$ as a solvent and tetramethylsilane (TMS) as an internal standard. Chemical shifts were reported in ppm units with use of $\delta$ scale. Mass spectra were scanned on a Shimadzu LCMS 2010 spectrometer. Anhydrous reactions were carried out in oven-dried glassware in nitrogen atmosphere.

\subsection{Antibacterial assay}

The newly synthesized compounds (5a-l) were screened for their antibacterial activity against Grampositive bacteria (Staphylococcus aureus (MTCC-96), Streptococcus pyogenes (MTCC-442)) and Gramnegative bacteria (Escherichia coli (MTCC-443), Pseudomonas aeruginosa (MTCC-1688). All MTCC cultures were collected from Institute of Microbial Technology, Chandigarh. The activity of compounds was determined as per National Committee for Clinical Laboratory Standards (NCCLS) protocol using Mueller Hinton Broth (Becton Dickinson, USA). ${ }^{25-28}$ Compounds were screened for their antibacterial activity as primary screening in six sets against Escherichia coli, Staphylococcus aureus, Pseudomonas aeruginosa and Streptococcus pyogenes at different concentrations of $1000,500,250 \mu \mathrm{g} / \mathrm{mL}$. The compounds found to be active in primary screening were similarly diluted to obtain $200,125,100,62.5,50,25$ and $12.5 \mu \mathrm{g} / \mathrm{mL}$ concentrations for secondary screening to test in a second set of dilution against all microorganisms. Inoculum size for test strain was adjusted to $10^{6} \mathrm{CFU} / \mathrm{mL}$ 
(Colony Forming Unit per milliliter) by comparing the turbidity (turbidimetric method). Mueller Hinton Broth was used as nutrient medium to grow and dilute the compound suspension for test bacteria. $2 \%$ DMSO was used as a diluent/vehicle to obtain the desired concentration of synthesized compounds and standard drugs to test upon standard microbial strains. Synthesized compounds were diluted to $1000 \mu \mathrm{g} / \mathrm{mL}$ concentration, as a stock solution. The control tube containing no antibiotic was immediately subcultured [before inoculation] by spreading a loopful evenly over a quarter of plate of medium suitable for the growth of test organisms. The tubes were then put for incubation at $37^{\circ} \mathrm{C}$ for $24 \mathrm{~h}$ for bacteria. $10 \mu \mathrm{g} / \mathrm{mL}$ suspensions were further inoculated on an appropriate media and growth was noted after $24 \mathrm{~h}$ and $48 \mathrm{~h}$. The highest dilution (lowest concentration) preventing appearance of turbidity was considered as minimum inhibitory concentration (MIC, $\mu \mathrm{g} / \mathrm{mL}$ ) i.e., the amount of growth from the control tube before incubation (which represents the original inoculum) was compared. A set of tubes containing only seeded broth and solvent controls were maintained under identical conditions so as to make sure that the solvent had no influence on strain growth. The result of this is greatly affected by the size of inoculum. The test mixture should contain $10^{6} \mathrm{CFU} / \mathrm{mL}$ organisms. Standard drug used in the present study was ampicillin for evaluating antibacterial activity which showed 100, 100, 250 and $100 \mu \mathrm{g} / \mathrm{mL}$ MIC against E. coli, $P$. aeruginosa, $S$. aureus and $S$. pyogenes, respectively.

\subsection{Antifungal assay}

The same compounds (5a-l) were tested for antifungal activity as primary screening in six sets against Candida albicans, Aspergillus niger and Aspergillus clavatus at various concentrations of 1000, 500, $250 \mu \mathrm{g} / \mathrm{mL}$. The compounds found to be active in primary screening were similarly diluted to obtain $200,125,100,62.5,50$ and $25 \mu \mathrm{g} / \mathrm{mL}$ concentrations for secondary screening to test in a second set of dilution against all microorganisms. Griseofulvin was used as a standard drug for antifungal activity, which showed 500, 100 and $100 \mu \mathrm{g} / \mathrm{mL}$ MIC against $C$. albicans, A. niger and A. clavatus. For fungal growth, in the present protocol, we have used Sabourauds dextrose broth at $28^{\circ} \mathrm{C}$ in aerobic condition for $48 \mathrm{~h}$.

\subsection{Statistical analysis}

Standard deviation value was expressed in terms of \pm $\mathrm{SD}$. On the basis of calculated value by using one-way
ANOVA method followed by independent two sample $t$ test, it was observed that differences below 0.001 level were considered as statistically significant. Compounds 5a-l were screened for their antibacterial and antifungal activities in six sets (n) against bacteria and fungi used in the present protocol.

\section{Results and discussion}

\subsection{Chemistry}

The Vilsmeier-Haack reaction on acetanilide provided a vital and efficient intermediate for the synthesis of several newer substituted heterocyclic compounds. The reaction was performed at $100^{\circ} \mathrm{C}$ for 4-16 h, using the typical Vilsmeiere-Haack reagent derived from phosphorus oxychloride and dimethylformamide. ${ }^{23}$ Although, the reaction proceeded uneventfully, the products formed were isolated using silica gel column chromatography. The reaction sequences employed for synthesis of the target compounds (5al) are illustrated in scheme 1 . The key chalcone intermediates $(\mathbf{2 a}-\mathbf{l})$ were synthesized through the ClaisenSchmidt condensation of equimolar amounts of acetophenone derivatives and chloroquinoline aldehyde (1) through stirring the reactants in aqueous ethanolic solution containing $20 \%$ sodium hydroxide at room temperature for $24 \mathrm{~h}$ in accordance with the method described in the literature. ${ }^{24}$

When 3-(2-chloroquinolin-3-yl)-1-(aryl)prop-2-en1-ones $(\mathbf{2 a}-\mathbf{l})$ were refluxed with guanidine nitrate in the presence of sodium hydroxide, 4-(aryl)-6-(2chloroquinolin-3-yl)pyrimidin-2-amines (3a-l) were formed. ${ }^{1} \mathrm{H}$ NMR spectrum of compound (3a) revealed a singlet characteristic peak for primary amine protons at $\delta=5.65 \mathrm{ppm}$ which was confirmed by disappearence of peak when exchange with $\mathrm{D}_{2} \mathrm{O}$. The IR spectra for (3a) showed the specific band for the primary amine at 3344 and $3450 \mathrm{~cm}^{-1}$. The ${ }^{13} \mathrm{C}$ NMR spectrum of the same compound revealed seventeen unequivalent carbons. Pyrimidine carbons displayed characteristic peaks at $\delta=103.5$ (C-5), 163.1 (C-2), 164.8 (C-6), 165.2 (C-4) ppm. Moreover, the mass spectrum of (5a) showed a molecular ion peak at $m / z=332\left(\mathbf{M}^{+}\right)$ corresponding to a molecular formula $\mathrm{C}_{19} \mathrm{H}_{13} \mathrm{ClN}_{4}$. Various substituted 2-chloro- $N$-(4-(2-chloroquinolin3-yl)-6-(aryl)pyrimidin-2-yl)acetamides (4a-l) were synthesized by electrophilic substitution reaction of chloroacetyl chloride with the corresponding parent 4-(aryl)-6-(2-chloroquinolin-3-yl)pyrimidin-2-amines (3a-l) in the presence of triethylamine as base and toluene as solvent. The structures of compounds 


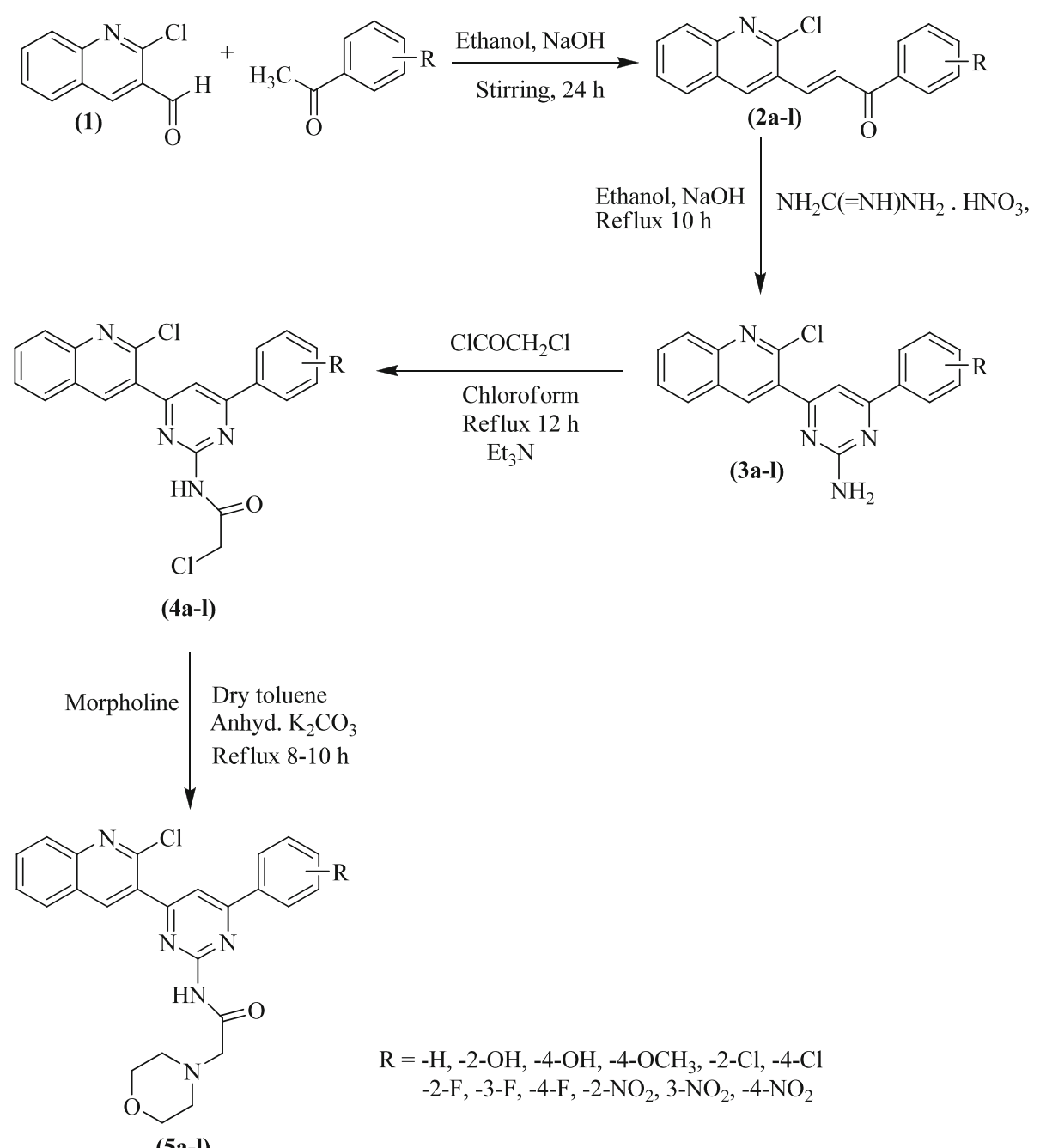

Scheme 1. Synthetic route for the preparation of title compounds (5a-1).

(4a-l) were determined by spectral data and elemental analysis, which gave the molecular formula (see Experimental). Then, condensation of 2chloro- $N$-(4-(2-chloroquinolin-3-yl)-6-(aryl)pyrimidin2-yl)acetamides (4a-l) with morpholine in the presence of anhydrous potassium carbonate furnished $N$-(4-(2-chloroquinolin-3-yl)-6-(aryl)pyrimidin-2-yl)-

2-morpholinoacetamides (5a-1). Structures of the isolated products $(\mathbf{5} \mathbf{a}-\mathbf{l})$ were confirmed on the basis of their spectral data. For example, the IR spectrum of compound (5a) revealed an absorption bands at 3314, 3230 and $1686 \mathrm{~cm}^{-1}$ characteristic to secondary amine and carbonyl groups, respectively. Absorption bands at 2962,2920 and $2850 \mathrm{~cm}^{-1}$ were due to stretching vibration corresponding to methylene group. Its ${ }^{1} \mathrm{H}$ NMR spectrum revealed two triplet signals at $\delta=$ 2.61 and $3.47 \mathrm{ppm}$ with coupling constants of nearly 4.5 and $4.8 \mathrm{~Hz}$ assignable to $\mathrm{N}\left(\mathrm{CH}_{2}\right)_{2}$ and $\mathrm{O}\left(\mathrm{CH}_{2}\right)_{2}$ of morpholine ring, respectively. Protons of methylene group dispalyed as a singlet at $\delta=3.91 \mathrm{ppm}$. Proton of secondary amine in amide linkage showed as a singlet at $\delta=10.21 \mathrm{ppm}$. Aromatic protons showed a multiplet centred on $\delta=7.34-8.47 \mathrm{ppm}$. The ${ }^{13} \mathrm{C}$ NMR spectrum of compound (5a) revealed twenty one unequivalent carbons. The carbonyl carbon was displayed at $\delta=173.1 \mathrm{ppm}$. Other important signals were displayed at $\delta=45.3$ and $67.3 \mathrm{ppm}$ for $\mathrm{N}\left(\mathrm{CH}_{2}\right)_{2}$ and $\mathrm{O}\left(\mathrm{CH}_{2}\right)_{2}$, respectively, while methylene carbon was appeared downfield at $\delta=63.2 \mathrm{ppm}$. Moreover, the mass spectrum of (5a) showed a molecular ion peak at $m / z=459\left(\mathbf{M}^{+}\right)$corresponding to a molecular formula $\mathrm{C}_{25} \mathrm{H}_{22} \mathrm{ClN}_{5} \mathrm{O}_{2}$.

\subsection{Biology}

4.2a Antimicrobial activity: Compounds (5a-l) were evaluated against Gram-positive, Gram-negative bacteria and fungi strains. The individual minimum inhibitory concentration (MIC, $\mu \mathrm{g} / \mathrm{mL}$ ) obtained for 


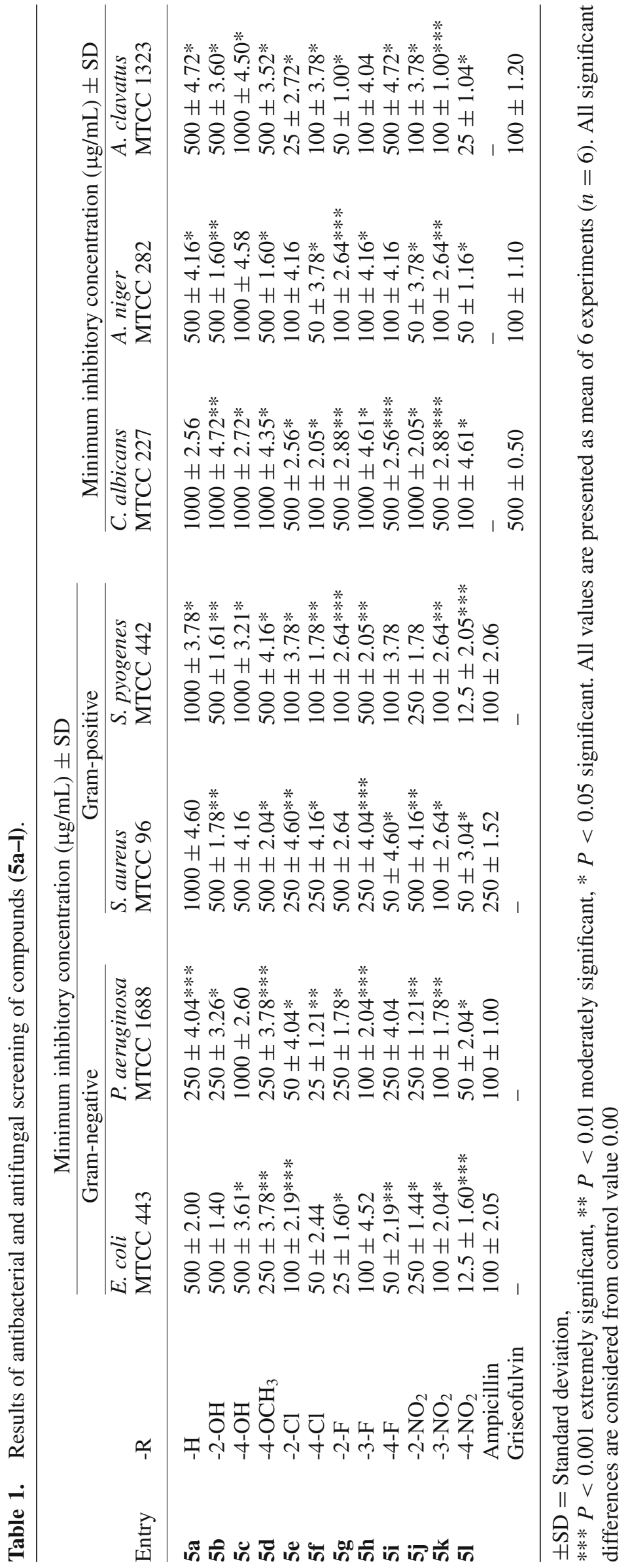


compounds (5a-l) are presented in table 1. It was observed that compounds $\mathbf{5 e}(2-\mathrm{Cl}), \mathbf{5 f}(4-\mathrm{Cl}), \mathbf{5 g}(2-\mathrm{F})$, $\mathbf{5 i}(4-\mathrm{F})$ and $\mathbf{5 l}\left(4-\mathrm{NO}_{2}\right)$ were most active compounds. From antibacterial screening results, it was observed that compounds $\mathbf{5 f}(4-\mathrm{Cl})$ and $\mathbf{5 i}(4-\mathrm{F})$ possessed good activity, whereas compounds $\mathbf{5 g}(2-\mathrm{F})$ and $\mathbf{5 l}\left(4-\mathrm{NO}_{2}\right)$ possessed excellent activity against $E$. coli. Compounds 5e $(2-\mathrm{Cl})$ and $\mathbf{5 l}\left(4-\mathrm{NO}_{2}\right)$ possessed very good activity against $P$. aeruginosa. When we replaced hydrogen in 5a by nitro and chloro group at fourth position of benzene ring, we got compound $\mathbf{5 f}$ which underwent enhancement in activity and possessed excellent activity against $P$. aeruginosa. Compound $\mathbf{5 k}\left(3-\mathrm{NO}_{2}\right)$ shown good activity, while compounds $\mathbf{5 i}(4-\mathrm{F})$ and $\mathbf{5 l}$ $\left(4-\mathrm{NO}_{2}\right)$ exhibited excellent activity against $S$. aureus. It was our observation that when we replaced hydrogen in $\mathbf{5 a}$ by $4-\mathrm{NO}_{2}$ led to compound $\mathbf{5 l}$ and they displayed excellent activity against $S$. pyogenes.

Antifungal activity showed that, compounds $5 f$ (4$\mathrm{Cl})$ and $\mathbf{5 l}\left(4-\mathrm{NO}_{2}\right)$ possessed excellent activity against C. albicans. Compounds $5 \mathbf{f}(4-\mathrm{Cl}), \mathbf{5 j}\left(2-\mathrm{NO}_{2}\right)$ and 5l $\left(4-\mathrm{NO}_{2}\right)$ possessed very good activity against $A$. niger. Compound $\mathbf{5 g}(2-\mathrm{F})$ possessed very good activity against $A$. clavatus. When nitro group was introduced at fourth position of benzene ring in 5a activity was enhanced and displayed excellent activity, while introduction of chloro group at second position of benzene ring in 5a displayed excellent activity against A. clavatus. Thus, we have discussed and compared antibacterial and antifungal activities based on standard drugs ampicillin and griseofulvin. Antimicrobial activity of compounds $\mathbf{5 e}, \mathbf{5 f}, \mathbf{5 g}, \mathbf{5 h}, \mathbf{5 i}, \mathbf{5 j}, \mathbf{5 k}$ and $\mathbf{5 l}$ tested against microbial strains by micro broth dilution assay is shown in figure 2 .

4.2b Structure-activity relationship: The results revealed that the activity was considerably affected by various substituents on the aromatic ring of pyrimidine nucleus. For antibacterial activity, it was observed that introduction of electron withdrawing group on benzene ring of pyrimidine nucleus showed considerable increase in antibacterial potency of compounds. It was observed from a result that compounds $\mathbf{5 g}$ and 5l containing fluoro and nitro substituents showed significant potency against Gram-negative bacteria E. coli. Amongst them, compound containing nitro group at fourth position on substituted benzene ring showed maximum inhibition against $E$. coli at MIC $12.5 \mu \mathrm{g} / \mathrm{mL}$. Compounds $\mathbf{5 i}$ and $\mathbf{5 l}$ containing flouro and nitro group showed good potency against $S$. aureus. Again compounds 51 containing nitro group at fourth position of benzene ring showed maximum inhibition against Gram-positive bacteria $S$. pyogenes. From the above results it was clear to note that electron withdrawing groups at fourth position of benzene ring showed maximum potency than electron releasing groups. All other compounds showed good to moderate potency. For antifungal activity, it has been observed that compounds containing chloro and nitro $\mathbf{5 f}$ and $\mathbf{5 l}$
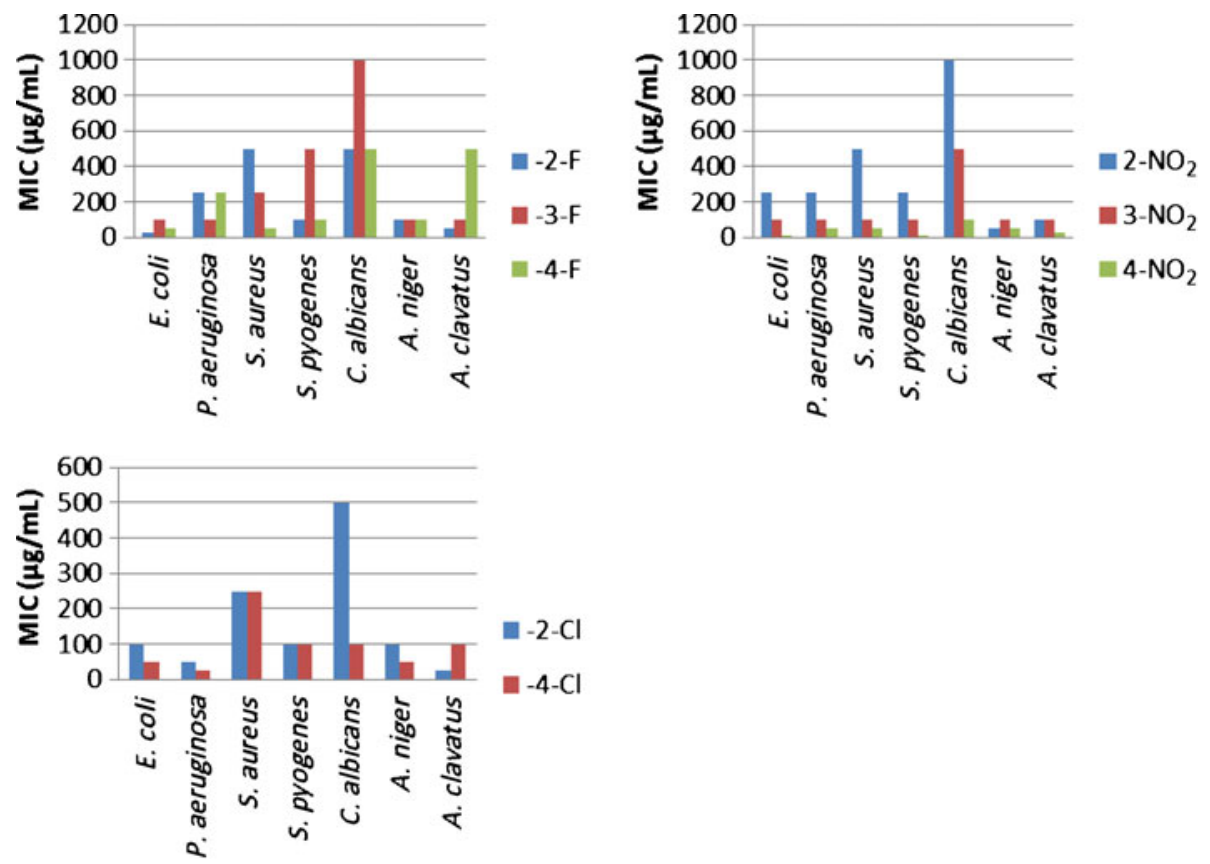

Figure 2. Antimicrobial activity of compounds $\mathbf{5 e}, \mathbf{5 f}, \mathbf{5 g}, \mathbf{5 h}, \mathbf{5 i}, \mathbf{5 j}, \mathbf{5 k}$ and $\mathbf{5 l}$ tested against microbial strains by micro broth dilution assay. 
showed excellent activity against $C$. albicans. Compounds 5e and 5l containing chloro and nitro group exhibited maximum potency against A. clavatus. It has been observed very strong correlation between antibacterial and antifungal activities.

\section{Conclusion}

The new compounds (5a-l) presented here clearly differ in their corresponding antimicrobial activity depending on the type of substituent in hybrid molecules. In the course of this study, particularly derivatives possessing electron withdrawing groups such as nitro and halogen are identified as exhibiting potential antibacterial activity against bacterial strains and antifungal activity against fungal strains. These results, combined with potential benefits or at least differences in pharmacokinetics make the titled quinoline, pyrimidine and morpholine congeners not only interesting simplified leads for the further chemical optimization of this class, but also potentially interesting for future scope to study their mechanism of action and would be worthy of additional structure-activity relationship investigation.

\section{Supplementary information}

The electronic supporting information can be seen in www.ias.ac.in/chemsci.

\section{Acknowledgement}

We thank the Department of Chemistry, Mahatma Gandhi Campus, Maharaja Krishnakumarsinhji Bhavnagar University, Bhavnagar for providing research and library facilities.

\section{References}

1. Lilienkampf A, Mao J, Wan B, Wang Y, Franzblau S G and Kozikowski A P 2009 J. Med. Chem. 522109

2. Nasveld P and Kitchener S 2005 Trans. R. Soc. Trop. Med. Hyg. 992

3. Leatham P A, Bird H A, Wright V, Seymour D and Gordon A 1983 Eur. J. Rheumatol. Inflamm. 6209

4. Denny W A, Wilson W R, Ware D C, Atwell G J, Milbank J B and Stevenson R J 2006 U.S Patent 7064117, June 20

5. Mahamoud A, Chevalier J, Davin-Regli A, Barbe J and Pages Jean-Marie 2006 Curr. Drug Targ. 7843

6. Muruganantham N, Sivakumar R, Anbalagan N, Gunasekaran V and Leonard J T 2004 Biol. Pharm. Bull. 271683

7. Maguire M P, Sheets K R, McVety K., Spada A P and Zilberstein A 1994 J. Med. Chem. 372129

8. Ahmed N, Brahmbhatt K G, Sabde S, Mitra D, Singh I P and Bhutani K K 2010 Bioorg. Med. Chem. 182872
9. Strekowski L, Mokrosz J L, Honkan V A, Czarny A, Cegla M T, Wydra R L, Patterson S E and Schinazi R F 1991 J. Med. Chem. 341739

10. Van Veldhoven J P D, Chang L C W, von Frijtag Drabbe Kunzel J K, Mulder-Krieger T, Struensee-Link R, Beukers M W, Brussee J and IJzerman A P 2008 Bioorg. Med. Chem. 16274

11. Hughes T V, Emanuel S L, Beck A K, Wetter S K, Connolly P J, Karnachi P, Reuman M, Seraj J, FuentesPesquera A R, Gruninger R H, Middleton S A, Lin R, Davis J M and Moffat D F C 2007 Bioorg. Med. Chem. Lett. 173266

12. Chhabria M T, Bhatt H G, Raval H G and Oza P M 2007 Bioorg. Med. Chem. Lett. 171022

13. Sayle K L, Bentley J, Boyle F T, Calvert A H, Cheng Y, Curtin N J, Endicott J A, Golding B T, Hardcastle I R, Jewsbury P, Mesguiche V, Newell D R, Noble M E M, Parsons R J, Pratt D J, Wang L Z and Griffin R J 2003 Bioorg. Med. Chem. Lett. 133079

14. Pastor A, Alajarin R, Vaquero J J, Alvarez-Builla J, Fau de Casa-Juana M, Sunkel C, Priego J G, Fonseca I and Sanz-Aparicio J 1984 Tetrahedron 508085

15. Youssouf M S, Kaiser P, Singh G D, Singh S, Bani S, Gupta V K, Satti N K and Suri K A 2008 Int. Immunopharmacol. 81049

16. Gasse C, Douguet D, Huteau V, Marchal G, MunierLehmann H and Pochet S 2008 Bioorg. Med. Chem. 16 6075

17. Govindaraju R, Gopalakrishnan M, Thanusu J and Kanagarajan V 2009 Med. Chem. Res. 18341

18. Ranise A, Bondavalli F, Bruno O, Schenone S, Losasso C, Costantino M, Cenicola M L, Donnoli D and Marmo E 1991 Farmaco 46317

19. Hale J J, Mills S G, MacCoss M, Dorn C P, Finke P E, Budhu R J, Reamer R A, Huskey S W, Luffer-Atlas D, Dean B J, McGowan E M, Feeney W P, Chiu S H L, Cascieri M A, Chicchi G G, Kurtz M M, Sadowski S, Ber E, Tattersall F D, Rupniak N M J, Williams A R, Rycroft W, Hargreaves R, Metzger J M and MacIntyre D E 2000 J. Med. Chem. 431234

20. Avramova P, Danchev N, Buyukliev R and Bogoslovova T 1998 Arch. Pharm. 331342

21. Desai N C, Rajpara K M and Joshi V V 2012 Bioorg. Med. Chem. Lett. 226871

22. Desai N C, Rajpara K M, Joshi V V, Vaghani H V and Satodiya H M 2012 Med. Chem. Res. doi:10.1007/ s00044-012-0121-z

23. Meth-Cohn O 1993 Heterocycles 35539

24. Elgazwy A S H 2008 Monatsh. Chem. 1391285

25. Desai NC, Rajpara K M, Joshi V V, Vaghani H V and Satodiya H M 2012 Anti-Infective Agents 1075

26. Desai N C, Joshi V V, Rajpara K M, Vaghani H V and Satodiya H M 2012 J. Fluor. Chem. 14267

27. National committee for clinical laboratory, Standards Methods for Dilution Antimicrobial Susceptibility Tests for Bacteria that grow aerobically approved standard, third ed. NCCLS Publication M7-A3, 1993 Villanova, PA

28. National committee for clinical laboratory standards, Reference Method for Broth Dilution Antifungal Testing of Yeasts, Proposed Standard. NCCLS Document M27-P; 1992 Villanova, PA 